\title{
ON SUBALGEBRAS OF SIMPLE LIE ALGEBRAS OF CHARACTERISTIC $p>0$
}

BY

\author{
B. WEISFEILER ${ }^{1}$
}

\begin{abstract}
The main results of the paper are Theorems I.5.1, II.1.3 and III.2.1. Theorem I.5.1 states that if a maximal subalgebra $M$ of a simple finite-dimensional Lie algebra $G$ has solvable quotients of dimension $\geqslant 2$, then every nilpotent element of $H$ acts nilpotently on $G$. Theorem II.1.3 states that if such a simple Lie algebra $G$ contains a maximal subalgebra which is solvable, then $G$ is Zassenbaus-Witt algebra. Theorem III.2.1 states that certain Z-graded finite-dimensional simple Lie algebras are either classical or the difference between the number of nonzero positive and negative homogeneous components is large.
\end{abstract}

In the present paper we study subalgebras of simple finite-dimensional Lie algebras in characteristic $p$. We assume throughout that the ground field is algebraically closed of characteristic $p \geqslant 7$. In the case $p=3$ there are counterexamples to some of our results. In characteristic 5 most proofs do not go through, and additional work does not seem to help.

In the study of simple Lie algebras in characteristic $p$ it is important to establish for each such an algebra $L$ that there exists in it a maximal sublagebra $L_{0}$ which defines a long filtration of $L$. This means that there is a nilpotent ideal $L_{1} \neq 0$ of $L_{0}$ such that $L_{1}$ acts nilpotently on $L$.

Our first aim is to establish that if $L_{0}$ does not define a long filtration, then $L_{0}$ must satisfy certain conditions. Our result in this direction is Theorem I.5.1 which implies, in particular, that if $L_{0}$ has solvable quotients of dimension $\geqslant 2$, then every nilpotent ideal of $L_{0}$ acts nilpotently on $L$. The proof of this result is based on results of $H$. Zassenhaus and $R$. Block about representations of Lie algebras and on ideas of V. Kac and I. L. Kantor about realizations of graded Lie algebras.

In trying to apply Theorem I.5.1 one has to consider the case when a maximal subalgebra $L_{0}$ has both nilpotent ideals and large solvable quotients. The simplest case which comes to mind where both these conditions hold is that of a solvable maximal subalgebra. Certain special cases of a solvable maximal subalgebra were considered by several people, among them by J. R. Schue [11] and M. I. Kuznetsov [9]. We heavily use results and ideas of the Kuznetsov paper. Because of the existence of a long filtration the question of classifying simple Lie algebras $L$ with a solvable maximal subalgebra $L_{0}$ is reduced to the question of classifying certain

Received by the editors April 23, 1982 and, in revised form, October, 1982.

1980 Mathematics Subject Classification. Primary 17B50.

' Partially supported by NSF.

O1984 American Mathematical Society $0002-9947 / 84 \$ 1.00+\$ .25$ per page 
graded simple Lie algebras whose zeroth term is solvable. This turns out to be rather hard. At any rate, the result is not surprising. It says that $L$ is of type $A_{1}$ or $W_{1}$. This is the main result, Theorem II.1.3.

Part III contains results about graded Lie algebras which are used in Part II but which seem to have wider applicability. They were also removed to streamline (to an extent) exposition in Part II. We also include in Part III some other results which seem to be in the spirit of that part. In particular, we include Theorem III.2.1 which states that a simple graded Lie algebra $G=\bigoplus_{i=-q}^{r} G_{i}$, satisfying certain additional conditions, is either classical (and then $q=r)$ or $\max (q, r) \geqslant((p-1) / 2) \min (q, r)$.

To conclude this account of the results of the present paper we would like to mention that the recent work of R. Block and R. Wilson has resulted in much more final structure results and will in the near future result in a classification of simple Lie $p$-algebras.

We do not give indications of proofs in this introduction because the paper is split into several parts which are fairly independent and straightforward. More precisely, Part II needs only one result from Part I; however, it uses several auxiliary results placed in Part III. Part III, the Appendix, is completely independent.

Notation and convention. We assume that the ground field $k$ is algebraically closed of characteristic $p \geqslant 7$. Our terminology is mostly standard. A Lie algebra $L$ over $k$ is classical if it is a direct sum of simple classical Lie algebras.

For subspaces $X, Y, Z, \ldots$ of $L$ we denote by $\langle X, Y, Z, \ldots\rangle$ the subalgebra of $L$ generated by $X, Y, Z, \ldots$. For subspaces $X, Y$ of $L$ such that $[X, Y] \subseteq Y$ we denote by $\operatorname{ad}_{Y} X$ the subspace of End $_{k} Y$ consisting of linear operators $y \rightarrow[x, y], x \in$ $X$; ad $X$ refers to the case $Y=L$. A subspace $X$ of $L$ is nil if $(\operatorname{ad} X)^{\operatorname{dim} L}=0$.

We are often led to consider divided power algebras. We denote by $B_{m}(F)$ the divided power algebra in $m$ variables associated to a flag $F$ (i.e., to a sequence of $m$ integers). In one or two variables we write

$$
B_{1}(n)=k\{x\}=\bigoplus_{0 \leqslant i<p^{n}} k x^{\{i\}}, \quad B_{2}(q, r)=k\{x, y\}=\bigoplus_{\substack{0 \leqslant i<p^{q} \\ 0 \leqslant j<p^{r}}} k x^{\{i\}} y^{\{j\}}
$$

The Lie algebra of special derivations of $B_{m}(F)$ is denoted $W_{m}(F)$. When we say that $L$ is of type $W_{1}$ it means that $L$ is isomorphic to $W_{1}(n)$ for some $n$. The rings $B_{m}(F)$ have ring filtration (by total degree, for example, $B_{1, m}(n)=\bigoplus_{m \leqslant i<p^{n}} k x^{\{i\}}$ ). This induces a filtration on $W_{m}(F)$ via $W_{m, j}(F)=\left\{x \in W_{m}(F) \mid x\left(B_{m, r}(n)\right) \subseteq\right.$ $B_{m, r+j}(n)$ for all $\left.r\right\}$. Automatically $W_{m}(F)=W_{m,-1}(F)$. We usually use $C$ to denote the maximal ideal of $B_{m}(F)$. Thus $C=B_{m .1}(F)$. Then $W_{m .0}(F)=\{x \in$ $\left.W_{m}(F) \mid x(C) \subseteq C\right\}$. Both $B_{m}(F)$ and $W_{m}(F)$ can be made into graded algebras via $B_{m}(F)=\oplus B_{m}(F)_{(i)}$, where $B_{m}(F)_{(i)}$ consists of homogeneous elements of total degree $i$. Then $W_{m}(F)_{(i)}=\left\{x \in W_{m}(F) \mid x\left(B_{m}(F)_{(\jmath)}\right) \subseteq B_{m}(F)_{(i+j)}\right.$ for all $\left.j\right\}$. This grading is called standard. If $m=1$, then $B_{1}(n)_{(i)}=k x^{\{i\}}$ and $W_{1}(n)_{(j)}=k e_{j}$, where $e_{j}=x^{\{j+1\}} \partial, j=-1,0, \ldots, \partial x^{\{j\}}=x^{\{j-1\}}$. In particular, $\left[e_{-1}, e_{j}\right]=e_{j-1}$, $\left[e_{0}, e_{j}\right]=j e_{j}$.

Finally, we record some terminology and notation, related to $\mathbf{Z}$-graded and filtered Lie algebras (see [12]). A Lie algebra $L$ with a sequence $\left\{L_{1}\right\}$ of subspaces is 
filtered if $L \supset \cdots \supset L_{-1} \supset L_{0} \supset L_{1} \supset \cdots$ and $\left[L_{i}, L_{j}\right] \subseteq L_{i+j}$. A Lie algebra $G=\oplus_{i=-q}^{r} G_{i}$ is graded if $\left[G_{i}, G_{j}\right] \subseteq G_{i+j}$. We call $q$ the depth and $r$ the height of the graded Lie algebra. If $L$ is filtered with filtration $L_{i}$ then the associated graded algebra is $G=\oplus G_{i}, G_{i}=L_{i} / L_{i+1}$, where, for $x \in L_{i}, y \in L_{j}$, we define $[x+$ $\left.L_{i+1}, y+L_{j+1}\right]=[x, y]+L_{i+j+1}$. This gives $G$ a structure of graded Lie algebra. If $G=\oplus G_{i}$ is a Z-graded Lie algebra, then $M(G)$ is the largest ideal of $G$ contained in $\bigoplus_{i \leqslant-2} G_{i}$. It is defined constructively as

$$
\begin{gathered}
M^{0}(G)=0, \quad M^{i+1}(G)=\left\{x \in \bigoplus_{i \leqslant-2} G_{i} \mid\left[x, \bigoplus_{i>0} G_{i}\right] \subset M^{i}(G)\right\}, \\
M(G)=\bigcup_{i} M^{i}(G) .
\end{gathered}
$$

It is easy to see that $M(G)$ inherits a grading from $G: M(G)_{i}=M(G) \cap G_{i}$, $M(G)=\oplus M(G)_{i}$. Next, $A(G)$ is the ideal of $G$ generated by $\bigoplus_{i<0} G_{i}$. It is also graded, $A(G)=\oplus A(G)_{i}, A(G)_{i}=A(G) \cap G_{i}$. We have $A(G)_{i}=G_{i}$ for $i<0$, $A(G)_{0}=\Sigma\left[G_{i}, G_{-i}\right]$, etc.

\section{PART I. MAXimal SUbalgebras OF Simple Lie algebraS}

The purpose of this part is to develop some techniques for working with Lie algebras when there is no long filtration. The main result says that a maximal subalgebra which has large solvable quotients cannot be too bad. Our main tools are representation-theoretic (after H. Zassenhaus and R. Block) and those developed by V. Kac and I. Kantor in their study of graded Lie algebras.

We start by outlining rudiments of the representation theory of Lie algebras in characteristic $p>0$ after $\mathbf{H}$. Zassenhaus. This permits us to naturally distinguish a class of subalgebras of a Lie algebra which we call "bad". In the case when a bad subalgebra has large solvable quotients we are able to arrive at a continuation using R. Block's theory of differentially irreducible modules together with rudiments of $I$. Kantor's realization of graded Lie algebras.

Recall that the ground field is algebraically closed of characteristic $p>5$.

1. Recollections on representations of Lie algebras (after H. Zassenhaus [16]). Let $G$ be a Lie algebra of dimension $n$ over $k$. Let $U(G)$ be the universal enveloping algebra of $G$, let $Z$ be its center, and let $Z_{0}$ (denoted $o$ in [16]) be the subalgebra of $Z$ generated by $k \cdot 1$ and by $Z \cap\left(G+k G^{p}+k G^{p^{2}}+\cdots\right)$. Set $G^{x}=\operatorname{Spec} Z_{0}$. In [16, p. 13 (top)] Zassenhaus establishes that $G^{x} \simeq \mathbf{A}^{n}$ (an $n$-dimensional affine space over $k$ ), and at the very end of the paper [16] he states that $G^{x}$ has a structure of a vector space over $\mathbf{F}_{p}$. The additive structure of $G^{x}$ is described by him as follows. A point $l \in G^{x}$ is an algebra homomorphism $l: Z_{0} \rightarrow k$. For every finite-dimensional $Z_{0}$-module $N$ we have a Fitting decomposition $N=\bigoplus_{l \in G^{x}} N(l)$, where $N(l)=\{n$

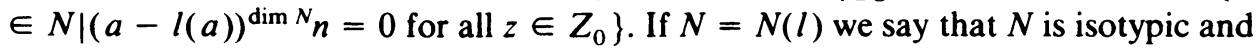
write $l_{N}$ for the corresponding $l$. Irreducible modules are automatically isotypic. By [16, Theorem 12 and subsequent discussion] we see that a tensor product of isotypic $Z_{0}$-modules $M$ and $N$ is isotypic and $l_{M \otimes N}=l_{M}+l_{N}$. Let us remark that every $G$-module can be considered as a $U(G)$-module and, therefore, by restriction as a 
$Z_{0}$-module. We call a $G$-module isotypic if it is isotypic as the $Z_{0}$-module. We record the results of [16] which were discussed above as

1.1. FACTs. (i) In the center $Z$ of $U(G)$ consider the subalgebra $Z_{0}$ generated by 1 and $Z \cap\left(\sum_{i=0}^{\infty} k G^{p^{i}}\right)$. Then $\operatorname{Spec} Z_{0}$ has a structure of a vector space over $F_{p}$.

(ii) For two isotypic G-modules $M$ and $N$ we have $l_{M \otimes N}=l_{M}+l_{N}$, where the addition is that of $G^{x}$.

1.2. Lemma. Consider $G$ as a G-module via adjoint action. Then $G$ is an isotypic $G$-module and $l_{G}=0$.

Proof. Let $G=\oplus_{l \in G^{x}} G(l)$ (a direct sum of $G$-modules). Then each $G(l)$ is a $G$-module and, therefore, an ideal of $G$. Evidently, $\left[G\left(l_{1}\right), G\left(l_{2}\right)\right] \subseteq G\left(l_{1}\right) \cap G\left(l_{2}\right)=$ 0 if $l_{1} \neq l_{2}$. On the other hand, by 1.1 (ii) we have $\left[G\left(l_{1}\right), G\left(l_{2}\right)\right] \subseteq G\left(l_{1}+l_{2}\right)$ whence $[G(l), G(l)]=0$ if $l \neq 0$. Thus $\tilde{G}=\oplus_{l \neq 0} G(l)$ is in the center of $G$ and $G=G(0) \oplus \tilde{G}$ (a direct sum of algebras). Let $M$ be an irreducible nontrivial module for $G(l), l \neq 0$. Make $M$ into a $G$-module by setting $G\left(l^{\prime}\right) M=0$ if $l \neq l^{\prime}$. By 1.1(ii) we have $l_{G(l) \otimes M}=l_{G(l)}+l_{M}=l+l_{M}$. On the other hand, the action of $G$ on $M$ determines a map $G \otimes M \rightarrow M$ which factors through $G(l) \otimes M$ as $M$ is a trivial $\oplus_{l^{\prime}+1} G\left(l^{\prime}\right)$-module. Thus we have $l_{G(l) \otimes M}=l_{M}$ whence $l=0$, a contradiction.

1.2.1. Remarks. (i) $M$ can be a module with trivial action as well.

(ii) A more explicit description of the group structure on $G^{x}$ would show directly that $l_{G}=0$. But we avoid this approach.

1.3. LeMMA. Let $n \in G$ be nilpotent and let $m$ be an integer such that $(\mathrm{ad} n)^{p^{m}}=0$. Then $n^{p^{m}} \in Z_{0}$.

Proof. Since ad $y$ can be written in $U(G)$ as ad $y=L_{y}-R_{y}$, where $L_{y}$ (resp., $R_{y}$ ) is the operator of left (resp., right) multiplication by $y$, and since $L_{y}$ and $R_{y}$ commute, we have by the binomial formula

$$
(\operatorname{ad} y)^{q}=\sum_{i=0}^{q}(-1)^{i}\left(\begin{array}{c}
q \\
i
\end{array}\right) L_{y}^{q-i} R_{y}^{i}=\sum_{i=0}^{q}(-1)^{i}\left(\begin{array}{c}
q \\
i
\end{array}\right) L_{y^{q-},} R_{y^{\prime}}
$$

In particular, $0=(\operatorname{ad} n)^{p^{m}}=L_{n^{p m}}-R_{n^{p m}}$ whence $n^{p^{m}} x=x n^{p^{m}}$ for any $x \in U(G)$. Thus $n^{p^{m}} \in Z$. But then $n^{p^{m}} \in Z \cap\left(\sum_{i=0}^{\infty} k G^{p^{\prime}}\right) \subseteq Z_{0}$, as claimed.

1.4. Corollaries. Let $M$ be a simple G-module, $n \in G,(\operatorname{ad} n)^{p^{m}}=0$.

(i) Then $n$ acts on $M$ as $\lambda \cdot \operatorname{Id}_{M}+$ (nilpotent operator). Denote this $\lambda$ by $\chi_{M}(n)$.

(ii) We have $l_{M}\left(n^{p^{m}}\right)=\left(\chi_{M}(n)\right)^{p^{m}}$. In particular, if $l_{M}\left(n^{p^{m}}\right)=0$, then $\chi_{M}(n)=0$, i.e., $n$ acts on $M$ as a nilpotent operator.

Proof. Since $n^{p^{m}} \in Z$, it acts as a scalar on $M$. Let $\lambda_{1_{m}}, \ldots, \lambda_{t}$, be the eigenvalues of $n$. We have, therefore, $\lambda_{1}^{p^{m}}=\lambda_{2}^{p^{m}}=\cdots=\lambda_{t}^{p^{m}}$, whence $\lambda_{1}=\lambda_{2}=\cdots=\lambda_{t}$ as claimed in (i). By definition of $l_{M}$ we know that $n^{p^{m}}-l_{M}\left(n^{p^{m}}\right)$ is nilpotent. Thus $l_{M}\left(n^{p^{m}}\right)=\left(\chi_{M}(n)\right)^{p^{m}}$. This proves (ii).

1.4.1. Remarks. (i) Lemma 1.3 and its corollaries enable us to use condition $l_{M}=0:$ if $l_{M}=0$, then every nilpotent element of $G$ acts on $M$ as a nilpotent transformation. 
(ii) Actually $\chi$, considered as a function on the set of nilpotents of $G$ suffices for our purposes. It obviously possesses the properties we need: $\chi_{M \odot N}=\chi_{M}+\chi_{N}$ (if $M$ and $N$ are "isotypic") and $\chi_{G}=0$. We went to the trouble of looking at $Z_{0}, l_{M}$, etc., only because it seems to be the proper generality.

(iii) In the case when $G$ is a $p$-algebra, the statements similar to those of the present section are discussed in a paper of V. Kac and the author [13]. However, even for $p$-algebras $l_{v}$ may have different meaning here and in [13].

2. Bad maximal subalgebras. Let $H$ be a subalgebra of a Lie algebra $G$. Let $U(H)$ be the universal enveloping algebra of $H$, and let $Z$ be the center of $U(H)$. Then we have Fitting decomposition

$$
G=\bigoplus_{\lambda \in \operatorname{Spec} Z} G_{\lambda}
$$

where $G_{\lambda}=\left\{y \in G \mid(a-\lambda(a))^{\operatorname{dim} G} y=0\right.$ for all $\left.a \in Z\right\}$. Let $\pi: \operatorname{Spec} Z \rightarrow \operatorname{Spec} Z_{0}$ $=H^{x}$ be the canonical projection induced by the embedding $Z_{0} \rightarrow Z$. Set $G(l)=$ $\oplus_{\pi(\lambda)-1} G_{\lambda}$.

2.1. Definition. A subalgebra $H$ of a Lie algebra $G$ is called bad if $G \neq G(0)$. (By Lemma $1.2, H$ is automatically a proper subalgebra.)

Our aim in this section is to prove

2.2. TheOREM. Let $G$ be a Lie algebra and let $H$ be its maximal subalgebra. Suppose that $G$ contains an ideal $\hat{G}$ such that

(a) $\hat{G}$ is a simple Lie algebra;

(b) any minimal ideal of $G$ coincides with $\hat{G}$;

(c) $G=\hat{G}+H$.

Suppose that $H$ is a bad subalgebra of $G$. Then there are $\mathrm{Z} / p$-gradings $G=$ $\oplus_{i \in \mathbf{Z} / p} G_{i}$ and $\hat{G}=\bigoplus_{i \in \mathbf{Z} / p} \hat{G}_{i}$ of $G$ and $\hat{G}$ such that $\hat{G}_{i} \subseteq G_{i}$ and $\oplus$

(i) $G_{0}=H, \hat{G}_{0}=H \cap \hat{G} \neq 0$;

(ii) $G_{i}=\hat{G}_{i}, G_{i} \neq 0$, for all $i \neq 0$;

(iii) $G_{i}, i \neq 0$, is an irreducible and faithful $G_{0}$-module;

(iv) $\left[\hat{G}_{i}, \hat{G}_{j}\right]=\hat{G}_{i+j}$ for all $(i, j) \neq(0,0)$.

(Note that if $G$ is simple then $G=\hat{G}$.)

Proof. By Fact 1.1(ii) we have $\left[G\left(l_{1}\right), G\left(l_{2}\right)\right] \subseteq G\left(l_{1}+l_{2}\right)$. Thus $G(0)$ is a subalgebra. We have $H \subseteq G(0)$ by Lemma 1.2 . Since $G \neq G(0)$ and since $H$ is maximal we have $H=G(0)$. Now take $l \neq 0$ such that $G(l) \neq 0$. Then $G(0)$ and $G(l)$ generate a subalgebra which is contained in $\oplus_{i \in \mathbf{Z} / p} G(i l)$. Since $H$ is maximal and $\oplus G(i l) \neq H$ we must have that $G=\oplus G(i l)$. Set $\hat{G}(i l)=G(i l) \cap \hat{G}$. Since $\hat{G}$ is an ideal we have $\hat{G}=\oplus \hat{G}(i l)$. Since $G=\hat{G}+H$ and $H \subseteq G(0)$ we have $\hat{G}(i l)=G(i l)$ for $i \neq 0$.

Now let $V$ be a simple $H$-submodule of $G(l)$. Consider the subalgebra $\tilde{G}$ of $G$ generated by $V$. Clearly, $\tilde{G} \subseteq \hat{G}$ (since $G(l)=\hat{G}(l) \subseteq \hat{G}$ ). On the other hand, $\tilde{G}$ is spanned by commutators $c\left(v_{1}, \ldots, v_{n}\right)=\left[v_{1}\left[v_{2} \cdots\left[v_{n-1}, v_{n}\right] \cdots\right]\right.$ with $v_{i} \in V$ and $n \in Z, n \geqslant 1$. We have $c\left(v_{1}, \ldots, v_{p}\right) \in G(0)=H$ for all choices of $v_{1}, \ldots, v_{p} \in V$. Therefore $c\left(v, v_{1}, \ldots, v_{p}\right)=\left[v, c\left(v_{1}, \ldots, v_{p}\right)\right] \in V$ for all $v \in V$ and all $v_{i} \in V, i=$ $1, \ldots, p$. Therefore $\tilde{G}$ is spanned by the $c\left(v_{1}, \ldots, v_{n}\right)$ with $v_{i} \in V, p \geqslant n \geqslant 1$. Now note that $H=G(0)$ normalizes $\tilde{G}$ and therefore $H+\tilde{G}$ is a subalgebra of $G$. Since $H$ 
is maximal we have $G=H+\tilde{G}$. Therefore $\tilde{G}$ is an ideal of $G$. By condition (b) we have $\hat{G} \subseteq \tilde{G}$, that is, $\tilde{G}=\hat{G}$. We can write the equality $\tilde{G}=\hat{G}$ as

$$
\hat{G}(i l)=[V[V \cdots[V, V] \cdots]](i \text { times }), \quad i \leqslant p,
$$

First apply (2.2.1) and get that $\hat{G}(l)=V$ so that $\hat{G}(l)$ is irreducible. Now consider (2.2.p). If $\hat{G}(0)=0$, then (2.2.p) says that $\hat{G}$ is nilpotent, a contradiction. So $\hat{G}(0) \neq 0$ and now (2.2.p) implies that $G(i l) \neq 0$ for all $i \in \mathbf{Z} / p$ and that $[G(-l), C(l)]=\hat{G}(0)$.

Since $G(i l) \neq 0$ for all $i$ we can apply the argument above to $i l, i \neq 0$, instead of $l$. This gives us that all $G(i l), i \neq 0$, are irreducible and $[G(i l), G(-i l)]=\hat{G}(0)$ for all $i \neq 0$.

Let us now show that $H$ is faithful on each $G(i l), i \neq 0$. Suppose that $\tilde{H}$ is the kernel of the action of $H$ on $G(l)$. Then $[\tilde{H}, G(l)]=0$ whence by $(2.2 . i), p \geqslant i>0$, we have that $[\tilde{H}, \hat{G}(i l)]=0$, that is, $\tilde{H}$ centralizes $\hat{G}$. Since $\tilde{H}$ is an ideal of $H$ and $G=H+\hat{G}$, we conclude that $\tilde{H}$ is an ideal of $G$. Thus $\tilde{H} \supseteq \hat{G}$, a contradiction (since $[\tilde{H}, \hat{G}]=0$ and $\hat{G}$ is simple).

Now we want to show that $[\hat{G}(i l), \hat{G}(j l)]=\hat{G}((i+j) l)$. First we remark that $[\hat{G}(0), G(i l)]=G(i l)$ for $i \neq 0$. Indeed, since $G(i l)$ is irreducible for $G(0)$, and $\hat{G}(0)$ is an ideal of $G(0)$, we have that $[\hat{G}(0), G(i l)]=0$ or $G(i l)$. The first case is impossible since $G(0)$ is faithful on $G(i l)$.

Next $(2.2 . *)$ applied to $j l$ instead of $l, p>j>0$, says that $[\hat{G}(j l), \hat{G}(m j l)]=$ $\hat{G}((m+1) j l)$ for all $0<m \leqslant p$. Since $j \neq 0$ we can take $m=i / j$, whence $[\hat{G}(j l), \hat{G}(i l)]=\hat{G}((i+j) l)$.

To conclude the proof we set $G_{i}=G(i l)$.

2.3. Remark. It should be rememberd that the indexing of $G_{i}$ depends on the choice of $l$. We shall use this freedom later.

2.4. Proposition. Let $H$ be a maximal sublalgebra of a Lie algebra $G$. Suppose that $H$ is bad. Then

(i) $G=\oplus_{i \in \mathbf{Z} / p} G($ il $)$ for some $l \in H^{x}$,

(ii) $G_{0}=H$;

(iii) $G(i l), i \neq 0$, are irreducible for $H$.

Proof of these statements is contained in the first part of the proof of Theorem 2.2.

2.5. Proposition. Let $H$ be a maximal subalgebra of a Lie algebra $G$. Suppose that $H$ is not bad. Then every nilpotent element of $H$ acts nilpotently on $G$. In particular, if $N$ is a nilpotent ideal of $H$, then $N$ acts nilpotently on $G$.

Proof. If $H$ is not bad, then $l_{G}=0$ (i.e. $G=G(0)$ ), whence our claim follows in view of Corollary 1.4(ii); see also Remark 1.4.1(i).

2.6. REMARK. The first step of the proof of Theorem 2.2 can be seen as obtaining a statement that a bad maximal subalgebra is the set of fixed points of a subgroup scheme isomorphic to $\mu_{p}$ ( $p$ th roots of unity) in the automorphism group scheme of $G$. For classical Lie algebras $G$ subgroups of Aut $G$ isomorphic to $\mu_{n}$ were classified 
by V. Kac in [7]. Of course, the fixed point subalgebras are not bad in this case, but nevertheless, the conclusions of Theorem 2.2 hold. One can also give a slight "generalization" of Theorem 2.2 as follows.

2.7. TheOREM. Let $B$ be a simple Lie algebra and let $\mathscr{H}$ be a subgroup scheme of multiplicative type in Aut $G$. If the subalgebra corresponding to the trivial character of $\mathscr{H}$ is maximal, then $\mathscr{H} \simeq \mu_{p}$ and the conclusions of Theorem 2.2 hold.

3. Generalities about graded Lie algebras (after V. Kac and I. Kantor). Our aim is to establish that certain maximal subalgebras of a simple Lie algebra $G$ cannot be bad. To this end we consider a $\mathbf{Z} / p$-graded simple Lie algebra $G=\bigoplus_{i \in \mathbf{Z} / p} G_{i}$ which is generated by its subspace $G_{-1} \oplus G_{0} \oplus G_{1}$ (actually, in our case, in view of Theorem $2.2, G$ is generated by $G_{-1}$ only). Such direct sum of spaces, satisfying natural conditions which automatically hold in our case, was called a local Lie algebra by V. Kac [5,6]. He used local Lie algebras to study Z-graded Lie algebras. In particular, he considered a Z-graded Lie algebra $\tilde{G}=\oplus \tilde{G}_{i}$ with $\tilde{G}_{i}=G_{i}$ for $i= \pm 1,0$, which is generated by $G_{-1} \oplus G_{0} \oplus \cdots$, but does not have graded ideals intersecting its local part. This construction of $\mathrm{V}$. Kac is a variant of the generators and relations description of Lie algebras. I. Kantor in [8] gave a realization of the algebra $\tilde{G}$. His realization is obtained by actually increasing the algebra $G$ to a certain universal algebra whose realization is quite easy to describe. However, to save space we do not give here the exposition of results of Kac and Kantor but just apply what is immediately useful for our purposes.

3.1. Consider again a $\mathbf{Z} / p$-graded simple Lie algebra $G=\oplus G_{i}$, satisfying the conclusions of Theorem 2.2. The map ad $x: G_{-1} \rightarrow G_{0}$ for $x \in G_{1}$ defines ad $x$ as an element of $\operatorname{Hom}_{k}\left(G_{-1}, G_{0}\right) \simeq G_{0} \otimes G_{-1}^{*}$. Thus we get a map $G_{1} \rightarrow G_{0} \otimes G_{-1}^{*}$. This map is injective for: (a) it is not zero because of Theorem 2.2(iv), and (b) its kernel is a $G_{0}$-submodule which must be zero in view of (a) above and Theorem 2.2(iii). The commutator between $G_{0} \otimes G_{-1}^{*}$ and $G_{-1}$ is given by $\left[g \otimes v^{*}, v\right]=v^{*}(v) g$ for $v \in G_{-1}$, $v^{*} \in G_{-1}^{*}$ and $g \in G_{0}$. We replace for a moment $G_{-1} \oplus G_{0} \oplus G_{1}$ by $G_{-1} \oplus G_{0} \oplus\left(G_{0}\right.$ $\otimes G_{-1}^{*}$ ) and we consider the free Lie algebra generated by this subspace modulo relations there; we call it $F$. We have: $F$ is $\mathrm{Z}$-graded, $F_{-1}=G_{-1}, F_{0}=G_{0}, F_{1}=G_{0} \otimes$ $G_{-1}^{*}$. On the other hand, $F_{-2}=\left[F_{-1}, F_{-1}\right]$ which means that $F_{-2}$ is a quotient of the $G_{0}$-module of the skew-symmetric square of $G_{-1}$. As $p \neq 2$ we can consider $F_{-2}$ as a quotient module of $G_{-1} \wedge G_{-1}$, where $G_{-1} \wedge G_{-1}=\left\{A \in G_{-1} \otimes G_{-1} \mid A^{t}=-A\right\}$, $(a \otimes b)^{t}=b \otimes a$. The commutator of $v_{1} \wedge v_{2} \in G_{-1} \wedge G_{-1}$ with $G_{0}$ is given by $\left[g, v_{1} \wedge v_{2}\right]=g v_{1} \wedge v_{2}+v_{1} \wedge g v_{2}$, and with $G_{0} \otimes G_{-1}^{*}$ it is given by $\left[g \otimes v^{*}, v_{1} \wedge\right.$ $\left.v_{2}\right]=v^{*}\left(v_{1}\right) g v_{2}-v^{*}\left(v_{2}\right) g v_{1}$. Indeed,

$$
\begin{aligned}
{\left[g \otimes v^{*},\left[v_{1}, v_{2}\right]\right] } & =\left[\left[g \otimes v^{*}, v_{1}\right] v_{2}\right]+\left[v_{1}\left[g \otimes v^{*}, v_{2}\right]\right] \\
& =\left[v^{*}\left(v_{1}\right) g, v_{2}\right]+\left[v_{1}, v^{*}\left(v_{2}\right) g\right]=v^{*}\left(v_{1}\right) g v_{2}-v^{*}\left(v_{2}\right) g v_{1} .
\end{aligned}
$$

Now returning back to our $G$ we identify $G_{1}$ with a $G_{0}$-submodule of $G_{0} \otimes G_{-1}^{*}$. Consider the subalgebra $\bar{G}$ of $F$ generated by $G_{-1} \oplus G_{0} \oplus G_{1}$. We have a surjective map $\bar{G} \rightarrow G$ which is an isomorphism on $G_{-1} \oplus G_{0} \oplus G_{1}$. Let $D_{-2} \subseteq F_{-2}$ be the intersection of the kernel of this map with $F_{-2}$. Since the map is injective on $G_{-1} \oplus G_{0} \oplus G_{1}$ we see that $D_{-2}=\left\{A \in F_{-2} \mid\left[G_{1}, A\right]=0\right\}$. 
We sum this up as

3.2. ThEOREM. Let $G=\oplus G_{i}$ be a graded Lie algebra satisfying the conclusions of Theorem 2.2. Then:

(i) $G_{1}$ can be identified with a $G_{0}$-submodule of $G_{0} \otimes G_{-1}^{*}$ with $\left[g_{1}, g_{2} \otimes v^{*}\right]=$ $\left[g_{1}, g_{2}\right] \otimes v^{*}+g_{2} \otimes g_{1} v^{*}$ and $\left[v, g \otimes v^{*}\right]=-v^{*}(v) g$ for $g, g_{1}, g_{2} \in G_{0}, v^{*} \in G_{-1}^{*}$ and $v \in G_{-1}$.

(ii) $G_{-2}$ can be identified with a quotient of $G_{-1} \wedge G_{-1}$ by $D_{-2}=\left\{A \in G_{-1} \wedge\right.$ $\left.G_{-1} \mid[x, A]=0 \forall x \in G_{1}\right\}$, where the commutator of $G_{-1} \wedge G_{-1}$ with $G_{0} \otimes G_{-1}^{*}$ is given by

$$
\left[g \otimes v^{*}, v_{1} \wedge v_{2}\right]=v^{*}\left(v_{1}\right) g v_{2}-v^{*}\left(v_{2}\right) g v_{1} \text {. }
$$

4. Generalities on ideals of the $G_{0}$-term of a graded Lie algebra. Consider a local Lie algebra of V. Kac, i.e., a direct sum $G_{-1} \oplus G_{0} \oplus G_{1}$ with $G_{0}$ a Lie algebra, $G_{ \pm 1}$ its modules, and with a map of $G_{0}$-modules [, ]: $G_{+1} \otimes G_{-1} \rightarrow G_{0}$. We assume that $\left[G_{-i}, x\right] \neq 0$ for $0 \neq x \in G_{i}, i= \pm 1$. We make one further

4.1. Assumption. $\left[G_{1}, G_{-1}\right]=G_{0}$.

Let $K$ be a proper ideal in $G_{0}$. For a $K$-module $V$ we denote by $V^{K}$ (resp., by $V_{K}$ ) the largest submodule (resp., quotient) of $V$ on which $K$ acts trivially. The surjective map [, ]: $G_{1} \otimes G_{-1} \rightarrow G_{0}$ of $G_{0}$-modules factors through $G_{0} \rightarrow G_{0} / K$ to give a surjective map $G_{1} \otimes G_{-1} \rightarrow G_{0} / K$. As $K$ acts trivially on $G_{0} / K$ we actually get a surjective map $\left(G_{1} \otimes G_{-1}\right)_{K} \rightarrow G_{0} / K$. Dualizing we get an injective map

$$
\left(G_{0} / K\right)^{*} \rightarrow\left(\left(G_{1} \otimes G_{-1}\right)^{*}\right)^{K}=\operatorname{Hom}_{K}\left(G_{1}, G_{-1}^{*}\right) .
$$

4.2. Corollaries. (i) $\operatorname{dim}\left(G_{0} / K\right) \leqslant \operatorname{dim} \operatorname{Hom}_{K}\left(G_{1}, G_{-1}^{*}\right)$.

(ii) If $G_{1}$ and $G_{-1}$ are simple $K$-modules then $\operatorname{dim}(G / K)=1$ and $G_{1} \simeq G_{-1}^{*}$ (as $G_{0}$-modules)

(iii) If $G_{1}$ and $G_{-1}$ are simple $G_{0}$-modules, then $\operatorname{dim}\left(G_{0} /\left[G_{0}, G_{0}\right]\right) \leqslant 1$. Moreover, $G_{1} \simeq G_{-1}^{*}$ if $G_{0} \neq\left[G_{0}, G_{0}\right]$.

Proof. (i) is a direct consequence of (4.1.1). In case (ii) we have $\operatorname{dim} \operatorname{Hom}_{K}\left(G_{1}, G_{-1}^{*}\right) \leqslant 1$, whence $\operatorname{dim}\left(G_{0} / K\right) \leqslant 1$. Since $K \neq G_{0}$ it follows that $\operatorname{dim}\left(G_{0} / K\right)=1$. But then $G_{0} / K$ is a trivial $G_{0}$-module. Thus the $G_{0}$-module map $G_{1} \otimes G_{-1} \rightarrow G_{0} / K$ determines a $G_{0}$-invariant pairing between $G_{1}$ and $G_{-1}$. This pairing is nonzero because $\left[G_{1}, G_{-1}\right]=G_{0}$ and it is nondegenerate because $G_{1}$ and $G_{-1}$ are simple $G_{0}$-modules. Finally, (iii) is a partial converse of (ii). Namely, set $K=\left[G_{0}, G_{0}\right]$. Then $G_{0} / K$ is a trivial $G_{0}$-module. Thus the image of $\left(G_{0} / K\right)^{*}$ in $\operatorname{Hom}_{K}\left(G_{1}, G_{-1}^{*}\right)$ is actually in $\operatorname{Hom}_{G_{0}}\left(G_{1}, G_{-1}^{*}\right)$. Since both $G_{1}$ and $G_{-1}$ are simple $G_{0}$-modules we have $\operatorname{dim} \operatorname{Hom}_{G_{0}}\left(G_{1}, G_{-1}^{*}\right) \leqslant 1$. Therefore $\operatorname{dim}\left(G_{0} / K\right) \leqslant 1$. If $\operatorname{dim}\left(G_{0} / K\right)=1$, then $\operatorname{Hom}_{G_{0}}\left(G_{1}, G_{-1}^{*}\right) \neq 0$, whence $G_{1} \simeq G_{-1}^{*}$.

5. On the derived series of a bad maximal subalgebra. The purpose of this section (and of the first part of this paper as well) is to establish

5.1. TheOREM. Let $G$ be a simple Lie algebra and let $H$ be a maximal subalgbra of G. If $H$ is bad, then $\operatorname{dim}(H /[H, H]) \leqslant 1$ and $[[H, H],[H, H]]=[H, H]$. (Thus, solvable quotients of $H$ are at most one-dimensional.) 
We know that $G$ is $\mathbf{Z} / p$-graded, $G=\bigoplus_{i \in \mathbf{Z} / p} G_{i}$, with $G_{0}=H$. We have certain freedom (cf. Remark 2.3) in choosing $G_{-1}$. Let us choose $G_{-1}$ so that $\operatorname{dim} G_{-1} \geqslant \operatorname{dim} G_{i}$, $i \neq 0$. Our strategy is to show that the assumption that $H$ has solvable quotients of dimension $\geqslant 2$ leads to a contradiction with the assumption $\operatorname{dim} G_{-1} \geqslant \operatorname{dim} G_{i}$, $i \neq 0$.

Denote $H^{\prime}=[H, H]$ and $H^{\prime \prime}=\left[H^{\prime}, H^{\prime}\right]$.

5.2. LEMMA. $\operatorname{dim}\left(H / H^{\prime}\right) \leqslant 1$.

Proof. This follows directly from Corollary 4.2(iii) and Theorem 2.2(iii) (Assumption 4.1 is satisfied in view of Theorem 2.2(iv)).

5.3. Proposition. Assume that $H \neq H^{\prime}$. Then $G_{-1}$ is an irreducible $H^{\prime}$-module.

Proof. Suppose $G_{-1}$ is not irreducible for $H^{\prime}$. Then it is differentially irreducible with one derivation $\left(\operatorname{as} \operatorname{dim}\left(H / H^{\prime}\right)=1\right)$. Therefore by R. Block [1, Theorem 2 or 2] we see that $G_{-1} \simeq U \otimes B_{n}$ as an $H$-module. Here $U$ is an irreducible $H^{\prime}$-module, $B_{n}=k\left[x_{1}, \ldots, x_{n}\right] /\left(x_{1}^{p}, \ldots, x_{n}^{p}\right), H^{\prime}$ acts trivially on $B_{n}$, and $B_{n}$ has no $H$-invariant ideals. By R. Ree [10] (explicit in M. I. Kuznetsov [9, Proposition 2.1]) $B_{n}$ can be identified with an algebra of divided power series in one variable, $B_{n} \simeq B_{1}(n)=$ $k\{x\}$, and the action of $H$ on $B_{n}$ respects the structure of divided powers.

Now' we look at $F_{-2}$ (in notation of $\$ 3.1$ ). Let $N=\operatorname{dim} U$ and let $u_{1}, \ldots, u_{N}$ be a basis of $U$. We have $F_{-2}=G_{-1} \wedge G_{-1}$ so that a generic element $b \in G_{-2}$ can be written as

$$
b=\sum_{\left(i, i^{\prime}\right)<\left(j, j^{\prime}\right)} b_{i, i^{\prime}, j, j^{\prime}}\left(u_{i^{\prime}} \otimes x^{\{i\}}\right) \wedge\left(u_{j^{\prime}} \otimes x^{\{j\}}\right),
$$

where $b_{i, i^{\prime}, j, j^{\prime}} \in k, 0 \leqslant i, j \leqslant p^{n}-1,1 \leqslant i^{\prime}, j^{\prime} \leqslant N$, and $<$ is the lexicographic order. For $b \in F_{-2}$ we set

$$
\min (b)=\min \left\{\left(i, i^{\prime}\right) \mid b_{i, i^{\prime}, j . j^{\prime}} \neq 0 \text { for some } j, j^{\prime}\right\} .
$$

5.3.1. Lemma. Let $D_{-2}$ be the same as in Theorem 3.2(ii), and let $k \overline{\{x\}}$ be the set of divided powers with zero constant term. Then

$$
D_{-2} \cap(U \otimes k \overline{\{x\}} \wedge U \otimes k \overline{\{x\}})=0 .
$$

Before proving this claim let us show how Proposition 5.3 follows from it. Since $G_{-2}=\left(G_{-1} \wedge G_{-1}\right) / D_{-2}$ it follows that $U \otimes k \overline{\{x\}} \wedge U \otimes k \overline{\{x\}}$ is mapped monomorphically into $G_{-2}$. Thus

$$
\operatorname{dim} G_{-2} \geqslant \operatorname{dim}(U \otimes k \overline{\{x\}} \wedge U \otimes k \overline{\{x\}})=\frac{1}{2} N\left(p^{n}-1\right)\left(N\left(p^{n}-1\right)-1\right) .
$$

On the other hand, by our choice of $G_{-1}$ we have $N p^{n}=\operatorname{dim} G_{-1} \geqslant \operatorname{dim} G_{-2}$. Thus we have $N p^{n} \geqslant \frac{1}{2} N\left(p^{n}-1\right)\left(N\left(p^{n}-1\right)-1\right)$. After elementary transformations this inequality becomes $p^{2 n}+1+1 / N \leqslant(2+3 / N) p^{n}$. This last inequality clearly does not hold if $p^{n} \geqslant 5$. Since $p>5$ is among our general assumptions, we have a contradiction. Thus it remains only to prove Lemma 5.3.1.

5.3.2. Write $H=k d \oplus H^{\prime}$. Let $\left\{g_{i}\right\}$ be a basis of $H^{\prime}$. By Corollary 4.2(iii) we know that $G_{1} \simeq G_{-1}^{\prime}$. By Theorem 3.2(i), we can consider $G_{1}$ as a submodule of 
$H \otimes G_{-1}^{*}$. Then we can write $y \in G_{1}$ as $y=d \otimes \psi(y)+\sum g_{i} \otimes \psi_{i}(y)$, where $\psi$ and the $\psi_{i}$ are linear maps from $G_{1}$ to $G_{-1}^{*}$. As $H / H^{\prime}$ is a trivial $H$-module, $\psi$ is an $H$-module map. As $G_{1} \simeq G_{-1}^{*}$ is irreducible, $\psi$ is an isomorphism. Set $v^{*}=\psi(y)$ and $\varphi_{i}=\psi_{i} \psi^{-1}$. Then $y=d \otimes v^{*}+\sum g_{i} \otimes \varphi_{i}\left(v^{*}\right)$, where $\varphi_{i}: G_{-1}^{*} \rightarrow G_{-1}^{*}$ are linear maps. Here $v^{*}$ is the element of $G_{-1}^{*}$ corresponding to $y \in G_{1}$ under $G_{1} \simeq G_{-1}^{*}$; the coefficient of $d$ is $v^{*}$ as it is $k d$ that determines the pairing between $G_{1}$ and $G_{-1}$.

As $H^{\prime}$ acts trivially on $k\{x\}, k\{x\}$ has no $H$-invariant ideals. As $H$ preserves the divided power structure of $k\{x\}, d$ acts on $k\{x\}$ as $a_{0} \partial+\sum_{i>0} a_{i} x^{\{i\}} \partial$, where $\partial x^{\{i\}}=x^{\{i-1\}}$ and $a_{0} \neq 0$. Therefore $d$ acts on $G_{-1}=U \otimes k\{x\}$ as $\sum_{i \geqslant 0} A_{i} \otimes x^{\{i\}} \partial$, where $A_{i} \in$ End $U, A_{0} \neq 0$, and $(A \otimes P(x) \partial)(u \otimes Q(x))=A u \otimes(P \cdot \partial Q)(x)$. Consider $V=U \otimes \sum_{i>0} k x^{\{i\}}$. Then $d^{m} V \subseteq A_{0} U \otimes 1+V$. Since $V+\sum_{m \geqslant 0} d^{m} V$ is $H$-invariant and $G_{-1}$ is $H$-irreducible we have $A_{0} U=U$, i.e., det $A_{0} \neq 0$.

The following lemma is formulated in a slightly more general form than is immediately necessary. We use notation $\tilde{H}$ for the subalgebra of operators on $U \otimes k\{x\}$ preserving the filtration by degree of $x$ in $U \otimes k\{x\}$.

5.3.3. LemMA. Let $y \in H \otimes G_{-1}^{*}\left(=F_{1}\right)$ be such that $y \equiv A \otimes \partial \otimes \varphi\left(v^{*}\right) \bmod \tilde{H} \otimes$ $G_{-1}^{*}$, where $A \in$ End $U, A \neq 0$, and $\varphi \in$ End $G_{-1}^{*}$. Let

$$
b=\sum_{\left(i, i^{\prime}\right)<\left(j, j^{\prime}\right)} b_{i, i^{\prime}, j, j^{\prime}}\left(u_{i^{\prime}} \otimes x^{\{i\}} \wedge \tilde{u}_{j^{\prime}} \otimes x^{\{j\}}\right) \in F_{-2}
$$

and suppose that all summands in the expression for $b$ are nonzero. Let $(m, q)=$ $\min (b)>0$. If $m>0$, then the lowest term (with respect to powers of $x$ ) in the expression for $[b, y]$ is

$$
\begin{aligned}
& -\left[\sum_{i^{\prime}<j^{\prime}} b_{m, i^{\prime}, m, j^{\prime}} \varphi\left(v^{*}\right)\left(u_{i^{\prime}} \otimes x^{\{m\}}\right) \cdot A u_{j^{\prime}}\right. \\
& \left.\quad-\varphi\left(v^{*}\right)\left(\sum_{\left(m, i^{\prime}\right)<\left(j, j^{\prime}\right)} b_{m, i^{\prime}, j, j^{\prime}}\left(u_{j^{\prime}} \otimes x^{\{j\}}\right)\right) \cdot A u_{i^{\prime}}\right] \otimes x^{\{m-1\}} .
\end{aligned}
$$

REMARKS. (a) The expression $\varphi\left(v^{*}\right)\left(\sum u_{j^{\prime}} \otimes x^{\{j\}}\right)$ means the value of $\varphi\left(v^{*}\right) \in G_{-1}^{*}$ on $\sum u_{j^{\prime}} \otimes x^{\{j\}} \in G_{-1}$.

(b) We do not rule out the case that the given expression (or even $[b, y]$ itself) is zero.

Proof. It is clear that the lowest term belongs to $U \otimes x^{\{m-1\}}$. Therefore this lowest term is contained in $\left[b, A \otimes \partial \otimes \varphi\left(v^{*}\right)\right]$, and furthermore it is contained in

$$
\begin{array}{r}
{\left[\sum_{\left(m, i^{\prime}\right)<\left(j, j^{\prime}\right)} b_{m, i^{\prime}, j, j^{\prime}}\left(u_{i^{\prime}} \otimes x^{\{m\}} \wedge u_{j^{\prime}} \otimes x^{\{j\}}\right), A \otimes \partial \otimes \varphi\left(v^{*}\right)\right]} \\
=-\sum_{\left(m, i^{\prime}\right)<\left(j, j^{\prime}\right)} b_{m, i^{\prime}, j, j^{\prime}}\left(\left[\varphi\left(v^{*}\right)\left(u_{i^{\prime}} \otimes x^{\{m\}}\right) \cdot A \otimes \partial, u_{j^{\prime}} \otimes x^{\{j\}}\right]\right. \\
\left.+\left[u_{i^{\prime}} \otimes x^{\{m\}}, \varphi\left(v^{*}\right)\left(u_{j^{\prime}} \otimes x^{\{j\}}\right) \cdot A \otimes \partial\right]\right) \\
=-\sum_{\left(m, i^{\prime}\right)<\left(j, j^{\prime}\right)} b_{m, i^{\prime}, j, j^{\prime}}\left(\varphi\left(v^{*}\right)\left(u_{i^{\prime}} \otimes x^{\{m\}}\right) \cdot A u_{j^{\prime}} \otimes x^{\{j-1\}}\right. \\
\left.-\varphi\left(v^{*}\right)\left(u_{j^{\prime}} \otimes x^{\{j\}}\right) \cdot A u_{i^{\prime}} \otimes x^{\{m-1\}}\right) .
\end{array}
$$


Collecting together the terms from $U \otimes x^{\{m-1\}}$, we readily see that they have the form claimed in Lemma 5.3.3.

5.3.4. Proof of Lemma 5.3.1 concluded. Set $Q=\left(\sum_{i>q} k \cdot A u_{i}\right) \otimes x^{\{m-1\}}+\sum_{j \geqslant m} U$ $\otimes x^{\{j\}}$. Then it follows from Lemma 5.3.3 that

$$
[b, y] \equiv \varphi\left(v^{*}\right)\left[\sum_{(m, q)<\left(j, j^{\prime}\right)} b_{m, q, j, j^{\prime}}\left(u_{j^{\prime}} \otimes x^{\{j\}}\right)\right] \cdot A u_{q} \otimes x^{\{m-1\}}(\bmod Q) .
$$

Now let $b \in D_{-2}$ be such that $m>0$ in $(m, q)=\min (b)$ (i.e. $b \in D_{-2} \cap(U \otimes k \overline{\{x\}}$ $\wedge U \otimes k \overline{\{x\}})$ ). Then $[b, y]=0$ since $b \in D_{-2}$, whence $[b, y] \equiv 0 \bmod Q$. Since $A$ is nondegenerate and $\varphi=\mathrm{Id} \in \operatorname{End} G_{-1}^{*}$ we get from the above expression for $[b, y] \bmod Q$ that $\sum_{(m, q)<\left(j, j^{\prime}\right)} b_{m, q, j, j^{\prime}}\left(u_{j^{\prime}} \otimes x^{\{j\}}\right)=0$, i.e., $b_{m, q . j, j^{\prime}}=0$ for $\left(j, j^{\prime}\right)>$ $(m, q)$. This contradicts the construction of $(m, q)=\min (b)$ This contradiction proves Lemma 5.3.1.

5.4. Proof of Theorem 5.1. It remains to prove that $H^{\prime \prime}=H^{\prime}$. Suppose that $H^{\prime \prime} \neq H^{\prime}$. Then there is an ideal $K$ of $H, H^{\prime} \supset K \supseteq H^{\prime \prime}$ such that $\operatorname{dim}(H / K)=2$, $H=k d+k h+K$ with $[d, h]=h \bmod K$, and $H^{\prime}=k h+K$.

5.4.1. As in 5.3.2 we write any element $y \in G_{1}$ in the form $y=d \otimes v^{*}+h \otimes$ $\tilde{\varphi}\left(v^{*}\right)+\sum \tilde{g}_{i} \otimes \tilde{\varphi}_{i}\left(v^{*}\right)$, where $v^{*} \in G_{-1}^{*}$ corresponds to $y \in G_{1}$ under the (fixed) isomorphism $G_{1} \simeq G_{-1}^{*}, \tilde{\varphi}, \tilde{\varphi}_{i} \in \operatorname{End} G_{-1}^{*}$, and $\left\{\tilde{g}_{i}\right\}$ is a basis of $K$.

For $x \in H$ we know that $[x, y]$ corresponds to $x v^{*} \in G_{-1}^{*}$ under the isomorphism $G_{1} \simeq G_{-1}^{*}$. We use this fact for $x=d, x=h$, and $x \in K$. We have

$$
[d, y] \equiv d \otimes d v^{*}+h \otimes \varphi\left(d v^{*}\right) \quad \bmod K \otimes G_{-1}^{*} .
$$

On the other hand, a direct computation gives

$$
[d, y] \equiv d \otimes d v^{*}+h \otimes \varphi\left(v^{*}\right)+h \otimes d \varphi\left(v^{*}\right) \bmod K \otimes G_{-1}^{*} .
$$

Therefore $\varphi\left(d v^{*}\right)=\varphi\left(v^{*}\right)+d \varphi\left(v^{*}\right)$ or

$$
\varphi\left(d v^{*}\right)-d \varphi\left(v^{*}\right)=\varphi\left(v^{*}\right) \text {. }
$$

Similarly, $[h, y] \equiv d \otimes h v^{*}+h \otimes \varphi\left(h v^{*}\right) \bmod K \otimes G_{-1}^{*}$ or, by direct computation, $[h, y] \equiv-h \otimes v^{*}+d \otimes h v^{*}+h \otimes h \varphi\left(v^{*}\right) \bmod K \otimes G_{-1}$. Thus $\varphi\left(h v^{*}\right)=-v^{*}+$ $h \varphi\left(v^{*}\right)$, or

$$
\varphi\left(h v^{*}\right)-h \varphi\left(v^{*}\right)=-v^{*} .
$$

Finally, $[x, y] \equiv d \otimes x v^{*}+h \otimes \varphi\left(x v^{*}\right)+K \otimes G_{-1}^{*}$ for $x \in K$ or by direct computation $[x, y] \equiv d \otimes x v^{*}+h \otimes x \varphi\left(v^{*}\right)+K \otimes G_{-1}^{*}$; that is,

$$
\varphi\left(x v^{*}\right)=x \varphi\left(v^{*}\right) \text { for } x \in K \text {. }
$$

The last equality says that $\varphi \in \operatorname{End}_{K}\left(G_{-1}^{*}\right)$.

5.4.2. By Proposition 5.3 we know that $G_{-1}$ is irreducible for $H^{\prime}=k h+K$. If $G_{-1}$ were irreducible for $K$, then $\varphi \in$ End $_{K} G_{-1}^{*}$ would be a scalar. Applying (5.4.1.2) to this case we get a contradiction: $0=-1$.

Thus $G_{-1}$ is reducible for $K$.

5.4.3. Since $G_{-1}$ is irreducible for $H^{\prime}=K+k h$ we see that $G_{-1}$ is differentially irreducible for $K$ with one derivation (namely, $h$ ). Therefore we can write (as in Proposition 5.3 and in view of $\mathrm{R}$. Block ([1, Theorem 2] or [2]) that $G_{-1} \simeq U \otimes B_{n}$ 
with an irreducible $K$-module $U$, and $K$ acting trivially on $B_{n}$. Moreover, we have $B_{n}=$ End $_{K}\left(G_{-1}\right)$. Therefore we have an action of $G / K$ on $B_{n}$. Since $G_{-1}$ is irreducible for $H^{\prime}$ we see that $B_{n}$ does not have $h$-invariant ideals. Therefore it does not have $G / K$-invariant ideals. In view of R. Ree [10] and R. Wilson [15] (explicitly stated in M. I. Kuznetsov [9, Proposition 2.1]) there are two possibilities:

(i) $B_{n}$ has a structure of a truncated divided power algebra in one variable, i.e., $B_{n} \simeq B_{1}(n)=k\{x\}$ and $G / K$ acts on $B_{n}$ by special derivations of $k\{x\}$. Recall that a derivation $D$ of $k\{x\}$ is special if $D x^{\{s\}}=x^{\{s-1\}} D x$.

(ii) $B_{n}$ has a structure of a truncated divided power algebra in two variables, i.e, $B_{n} \simeq B_{2}(q, r)=k\{\tilde{x}, \tilde{y}\}$ with $q+r=n, q \geqslant r>0, G / K$ acts on $B_{n}$ by special derivations of $k\{\tilde{x}, \tilde{y}\}$, and there are two linearly independent derivations in $G / K$ which do not preserve filtration. Recall that a derivation $D$ of $k\{\tilde{x}, \tilde{y}\}$ is special if $D z^{\{s\}}=z^{\{s-1\}} D z$ for $z=\tilde{x}$ or $\tilde{y}$.

5.4.4. Let us look at the first case first. Let $\partial$ be the derivation of $k\{x\}$ given by $\partial x^{\{i\}}=x^{\{1-1\}}$. Then $h$ acts on $\operatorname{End}_{K} G_{-1}$ as $H \partial$ and $d$ acts as $D \partial$ with $H, D \in k\{x\}$. Also we represent $\varphi \in$ End $_{K} G_{-1}^{*} \simeq$ End $_{K} G_{-1} \simeq k\{x\}$ by $F \in k\{x\}$. Write $H=$ $\sum h_{i} x^{\{i\}}, D=\sum d_{i} x^{\{i\}}$ and $F=\sum f_{i} x^{\{i\}}$ with $h_{i}, d_{i}, f_{i} \in k$.

Since End ${ }_{K} G_{-1}$ has no $h$-invariant nontrivial ideals, the constant term of $H$ must be nontrivial, $h_{0} \neq 0$. Replacing $d$ by $d-\left(d_{0} / h_{0}\right) h$ we have that $d_{0}=0$ (this change causes also changes in $\varphi$ and the $\varphi_{i}$ ).

The relation $[d, h]=h$ (valid for $d, h$ as operators on $\operatorname{End}_{K} G_{-1}$ ) becomes $D \cdot \partial H-H \cdot \partial D=H$. Therefore $-h_{0} \cdot d_{1}=h_{0}$ whence $d_{1} \neq 0$. Now the relations (5.4.1.1), (5.4.1.2) become $[d, \varphi]=-\varphi,[h, \varphi]=$ Id or, by definition of the action of $G / K$ on End $_{K} G_{-1}^{*}, d(F)=-F, h(F)=1$. Thus we have $D \cdot \partial F=-F, H \cdot \partial F=1$. The first equality implies that $f_{0}=0$ (because $d_{0}=0$ ), and the second one that $f_{1} \neq 0$ (or, more precisely, $h_{0} \cdot f_{1}=1$ ).

Now, as in 5.3.2 we can write $y \in G_{1}$ in the form $y \equiv A \otimes \partial \otimes \varphi\left(v^{*}\right) \bmod \tilde{H} \otimes$ $G_{-1}^{*}$, where $\tilde{H}$ is the algebra of operators preserving the filtration of $U \otimes k\{x\} ; h$ acts on $G_{-1}=U \otimes k\{x\}$ as $\sum A_{i} \otimes x^{\{i\}} \partial$ with $A=A_{0}$, det $A \neq 0$ (this is because $d_{0}=0$, so $d$ preserves the filtration). By Lemma 5.3. and the notation of 5.3.4 we see that for $b \in D_{-2}$ with $m>0$ in $(m, q)=\min (b)$ we still have

$$
\varphi\left(v^{*}\right)\left(\sum_{(m, q)<\left(j, j^{\prime}\right)} b_{m, q, j, j^{\prime}}\left(u_{j^{\prime}} \otimes x^{\{j\}}\right)\right)=0
$$

(we use the fact that $A$ is still nondegenerate). (However, now $\varphi$ is not an isomorphism!) We have $\varphi\left(G_{-1}^{*}\right)=F \cdot G_{-1}^{*}=U^{*} \otimes(F)$, where $(F)=F \cdot k\{x\}$. Since $f_{0}=0, f_{1} \neq 0$ we have $f_{1}^{-1} F x^{\{i\}}=x^{\{i+1\}}+$ (higher terms) whenever $p+i+1$. Therefore $\operatorname{dim}(F) \geqslant p^{n}-p^{n-1}$. Let $M$ be a complement to $(F)^{\perp}$ in $k\{x\}$ and $V=M \cap k \overline{\{x\}}$. Then $\operatorname{dim} M \geqslant p^{n}-p^{n-1}, \operatorname{dim} V \geqslant p^{n}-p^{n-1}-1$. Now for $b \in$ $U \otimes V \wedge U \otimes V$ we have $\sum_{(m, q)<\left(j, j^{\prime}\right)} b_{m, q . j, j^{\prime}}\left(u_{j^{\prime}} \otimes x^{\{j\}}\right) \in U \otimes V$ and therefore if, in addition, $b \in D_{-2}$, we have $\varphi\left(G_{-1}^{*}\right)\left(\sum_{(m, q)<\left(j . j^{\prime}\right)} b_{m, q . j . j^{\prime}}\left(u_{j^{\prime}} \otimes x^{\{j\}}\right)\right)=0$. By our choice of $V$ this implies that $\sum_{(m, q)<\left(j . j^{\prime}\right)} b_{m . q . j . j^{\prime}}\left(u_{j^{\prime}} \otimes x^{\{j\}}\right)=0$, contradicting, as in 5.3.4, the definition of $\min (b)$. Thus $D_{-2} \cap(U \otimes V \wedge U \otimes V)=0$. Then $U \otimes V \wedge$ $U \otimes V$ is mapped injectively into $G_{-2}$. Therefore 


$$
\begin{aligned}
\operatorname{dim} G_{-2} & \geqslant \operatorname{dim}(U \otimes V \wedge U \otimes V) \\
& \geqslant \frac{1}{2} N\left(p^{n}-p^{n-1}-1\right) \cdot\left(N\left(p^{n}-p^{n-1}-1\right)-1\right),
\end{aligned}
$$

where $N=\operatorname{dim} U$. Since $N \cdot p^{n}=\operatorname{dim} G_{-1} \geqslant \operatorname{dim} G_{-2}$ we get the inequality $N p^{n}$ $\geqslant \frac{1}{2} N\left(p^{n}-p^{n-1}-1\right) \cdot\left(N\left(p^{n} p^{n-1}-1\right)-1\right)$ which, after multiplying through and collecting terms, becomes

$$
2 p^{2 n-1}+(2+3 / N) p^{n} \geqslant p^{2 n}+p^{2 n-2}+(2+1 / N) p^{n-1}+1+1 / N
$$

The left-hand side is clearly bounded from above by $7 p^{2 n-1}$. Therefore for $p \geqslant 7$ the inequality is violated. This contradiction proves the theorem in case (i).

5.4.5. Let us look at the second case. So $\operatorname{End}_{K} G_{-1} \simeq B_{2}(q, r)=k\{\tilde{x}, \tilde{y}\}$ with $q+r=n, q \geqslant r>0$. Let $\partial_{1}, \partial_{2}$ be the derivations of $k\{\tilde{x}, \tilde{y}\}$ defined by $\partial_{1} \tilde{x}^{\{i\}}=$ $\tilde{x}^{\{i-1\}}, \partial_{1} \tilde{y}=0, \partial_{2} \tilde{x}=0, \partial_{2} \tilde{y}^{\{j\}}=\tilde{y}^{\{j-1\}}$. Since End ${ }_{K} G_{-1}$ has no $h$-invariant ideals we see that $h$ acts on $k\{\tilde{x}, \tilde{y}\}$ as $a \partial_{1}+b \partial_{2}+$ (higher terms) with $a \partial_{1}+b \partial_{2} \neq 0$. If $a \neq 0$ we can replace $\tilde{y}$ by $b \tilde{x}-a \tilde{y}$ and thereby achieve that $h$ acts on $k\{\tilde{x}, \tilde{y}\}$ as $\partial_{1}+$ (higher terms). Otherwise, $h$ acts as $b \partial_{2}+$ (higher terms), $b \neq 0$. To simplify our notation let us set $x=\tilde{x}, y=\tilde{y}$ in the first case and $x=\tilde{y}, y=\tilde{x}$ in the second one. Then $\operatorname{End}_{K} G_{-1}=k\{x, y\}$ and $h$ acts on it as $c \partial x+$ (higher terms) with $c \neq 0$. We can write $d=D_{1} \partial_{x} \oplus+D_{2} \partial_{y}$ and $h=H_{1} \partial_{x}+H_{2} \partial_{y}$, where $D_{i}=\sum d_{i j k} x^{\{j\}} y^{\{k\}}$ and $H_{i}=\sum h_{i j k} x^{\{j\}} y^{\{k\}}$. We have by the above that $h_{100} \neq 0, h_{200}=0$. Replacing $d$ by $d-\left(d_{100} / h_{100}\right) h$ we have that $d_{100}=0$. By the remark at the end of $\S 2$ of [9] we have $d_{200} \neq 0$. To $\varphi \in$ End $_{K} G_{-1}^{*}$ we put into correspondence $F \in k\{x, y\}, F=$ $\sum f_{i j} x^{\{i\}} y^{\{j\}}$.

The conditions (5.4.1.1) and (5.4.1.2) become $d(F)=-F, h(F)=1$, or explicitly $D_{1} \partial_{x} F+D_{2} \partial_{y} F=-F, H_{1} \partial_{x} F+H_{2} \partial_{y} F=1$. Looking at the constant term of $F$ in these expressions we get $d_{200} f_{01}=-f_{00}$ and $h_{100} f_{10}=1$. Thus either $f_{00} \neq 0$ (and then $\varphi$ is invertible) or $f_{00}=f_{01}=0, f_{10} \neq 0$. Write $d \equiv A \otimes \partial_{y} \bmod \tilde{H}$ and $h \equiv B \otimes$ $\partial_{x} \bmod \tilde{H}$ for $d$ and $h$ acting on $G_{-1}=U \otimes k\{x, y\}$. Here $\tilde{H}$ is the algebra of operators preserving filtration of $U \otimes k\{x, y\}$ and $A, B \in$ End $U$. Since $G_{-1}$ is irreducible for $H^{\prime}=k h+K$ the same argument as in $\$ 5.3 .2$ (with $d$ replaced by $h$ ) gives that det $B \neq 0$.

Now we can write any element $z \in G_{1}$ in the form $z=A \otimes \partial_{y} \otimes v^{*}+B \otimes \partial_{x} \otimes$ $\varphi\left(v^{*}\right) \bmod \tilde{H} \otimes G_{-1}^{*}$, det $B \neq 0, v^{*} \in G_{-1}^{*}$. Then any element from $G_{-1}$ has the form $\sum u_{i j} x^{\{i\}} y^{\{j\}}$ and an element $b \in F_{-2}=G_{-1} \wedge G_{-1}$ has the form

$$
b=\sum_{(i, j, l)<\left(i^{\prime}, j^{\prime}, l^{\prime}\right)} b_{i, j, l, i^{\prime}, j^{\prime}, l^{\prime}} u, \otimes x^{\{i\}} y^{\{j\}} \wedge u_{l^{\prime}} \otimes x^{\left\{i^{\prime}\right\}} y^{\left\{j^{\prime}\right\}}
$$

where $u_{i}, i=1, \ldots, N=\operatorname{dim} U$, is a basis of $U$ and the triples of integers are ordered lexicographically. Let

$$
\min (b)=\min \left\{(i, j, l) \mid b_{i, j, l, i^{\prime}, j^{\prime}, l^{\prime}} \neq 0 \text { for some } i^{\prime}, j^{\prime}, l^{\prime}\right\} .
$$

Write $\min (b)=(s, t, r)$. Then, as in Lemma 5.3.3, if $s>0$ then the lowest term 
(with respect to powers of $x$ and $y$ ) in $[b, z]$ is

$$
\begin{aligned}
& {\left[\varphi\left(v^{*}\right)\left(\sum_{(s, t, l)<\left(i^{\prime}, j^{\prime}, l^{\prime}\right)} b_{s, t, l, i^{\prime}, j^{\prime}, l^{\prime}}\left(u_{l^{\prime}} \otimes x^{\left\{i^{\prime}\right\}} y^{\left\{j^{\prime}\right\}}\right)\right) B u_{l}\right.} \\
& \left.\quad-\sum_{l<l^{\prime}} b_{s, t, l, s, t, l^{\prime}} \varphi\left(v^{*}\right)\left(u_{l} \otimes x^{\{s\}} y^{\{t\}}\right) B u_{l}\right] \otimes x^{\{s-1\}} y^{\{t\}} .
\end{aligned}
$$

Setting $Q=\sum_{i>r} k \cdot B u_{i} \otimes x^{\{s-1\}} y^{\{t\}}+\sum_{(i, j) \geqslant(s, t)} U \otimes x^{\{i\}} y^{\{j\}}$ we see as in 5.3.4 that

$$
[b, z] \equiv \varphi\left(v^{*}\right)\left(\sum_{(s, t, r)<(i, j, l)} b_{s, t, r, i, j, l}\left(u_{l^{\prime}} \otimes x^{\{i\}} y^{\{j\}}\right)\right) B u_{r} \otimes x^{\{s-1\}} y^{\{t\}}(\bmod Q) \text {. }
$$

Thus if $b \in D_{-2}, s>0$ in $(s, t, r)=\min (b)$, then $\lambda\left(b, v^{*}\right) B u_{r}=0$ for all $v^{*} \in G_{-1}^{*}$, where

$$
\lambda\left(b, v^{*}\right)=\varphi\left(v^{*}\right)\left(\sum_{(s, t, r)<(i, j, l)} b_{s, t, r, i, j, l}\left(u_{l} \otimes x^{\{i\}} y^{\{j\}}\right)\right) .
$$

Since det $B \neq 0$ it follows that $\lambda\left(b, v^{*}\right)=0$ for all $v^{*} \in G_{-1}^{*}$. Now, as in 5.4.4, $\varphi\left(G_{-1}^{*}\right)=U^{*} \otimes(F), F \in k\{x, y\}$. If $F$ is invertible, then $(F)=k\{x, y\}$ and $\varphi\left(G_{-1}^{*}\right)=G_{-1}^{*}$. Therefore in this case, $\lambda\left(b, v^{*}\right)=0$ for all $v^{*} \in G_{-1}^{*}$ is equivalent to $\sum_{(s, t, r)<(i, j, l)} b_{s, t, r, i, j, l}\left(u_{l} \otimes x^{\{i\}} y^{\{j\}}\right)=0$ in contradiction with the assumption $s>0$. Thus in this case $D_{-2} \cap(U \otimes K\{\overline{x, y}\} \wedge U \otimes k\{\overline{x, y}\}\}=0$, where $k\{\overline{x, y}\}=\{P$ $\left.\in K\{x, y\} \mid P=\sum_{i \geqslant 1} p_{i j} x^{\{i\}} y^{\{j\}}\right\}$.

If $F$ is not invertible, then $f_{00}=f_{01}=0$ and $f_{10} \neq 0$. As in 5.4.4 this implies that $\operatorname{dim}(F) \geqslant p^{n}-p^{n-1}$. Again as in 5.4 .4 we take $M$ to be a complement of $(F)^{\perp}$ in $k\{x, y\}$ and set $V=M \cap k\{\overline{x, y}\}$. For $b \in U \otimes V \wedge U \otimes V$ we have $\sum_{(s, t, r)<(i, j, l)} b_{s, t, r, i, j, l}\left(u, \otimes x^{\{i\}} y^{\{j\}}\right) \in U \otimes V$ and if, in addition, $b \in D_{-2}$, then $\lambda\left(b, v^{*}\right)=0$. By our choice of $V$ this implies a contradiction with a definition of $\min (b)$.

We have $\operatorname{dim} k\{\overline{x, y}\}=\operatorname{dim} k\{x, y\}-\operatorname{dim} k\{y\} \geqslant p^{n}-p^{n-1}$ and therefore $\operatorname{dim} V \geqslant p^{n}-2 p^{n-1}$. Since $D_{-2} \cap(U \otimes V \wedge U \wedge V)=0$ (we take $V=k\{\overline{x, y}\}$ if $F$ is invertible), we see that

$$
\operatorname{dim} G_{-2} \geqslant \operatorname{dim}(U \otimes V \wedge U \otimes V) \geqslant \frac{1}{2} N\left(p^{n}-2 p^{n-1}\right) \cdot\left[N\left(p^{n}-2 p^{n-1}\right)-1\right] .
$$

As before, we have (by our choice of $G_{-1}$ ) the inequality $\operatorname{dim} G_{-1} \geqslant \operatorname{dim} G_{-2}$ which becomes $2 N p^{n} \geqslant N^{2}\left(p^{2 n}-4 p^{2 n-1}+4 p^{2 n-2}\right)-N\left(p^{n}-2 p^{n-1}\right)$. We divide it by $N^{2} p^{n-1}$ and collect:

$$
4 p^{n}+\frac{3}{N} p \geqslant p^{n+1}+4 p^{n-1}+\frac{2}{N} .
$$

As $n \geqslant 2$ and $p \geqslant 7$ the right-hand side is $\geqslant 7 p^{n}+4 p$, which is larger than the left-hand side. This contradiction concludes the proof of Theorem 5.1.

5.5. Remarks and comments on the proof.

5.5.1. We derived a contradiction from $\operatorname{dim} G_{-2}>\operatorname{dim} G_{-1}$. Probably a more general fact takes place: If $p>5$ and if $G=\oplus_{i \in \mathbf{Z}} G_{i}$ is such that $G_{0}$ has solvable 
quotients of dimension $\geqslant 2$ and $\left[G_{1}, G_{-1}\right]=G_{0}$ with $G_{1}$ and $G_{-1}$ irreducible, then $G$ is infinite-dimensional (maybe even of exponential growth).

What we did was probably just verifying the first step.

5.5.2. In characteristic 3 there exist graded algebras of the type we rejected; see Frank [4].

\section{PART II. Solvable MaXimal SUbalgebras}

In this part we consider simple Lie algebras which contain a solvable maximal subalgebra. Using the results of the preceding part we conclude that such a subalgebra gives rise to a long filtration. We pass then to the graded Lie algebra and work hard to prove that certain graded Lie algebras with solvable zeroth term are of type $A_{1}$ or $W_{1}$. Our main tools are papers $[5,9,12]$.

The steps of the proof are the following: We consider a minimal counterexample $S=\oplus_{i=-q}^{r} S_{i}$. We show by constructing different smaller subalgebras that $S$ is generated by $S_{-1}$ and $S_{1}$ and then that $S_{1}$ is irreducible. This latter step required very detailed study of the structure of our algebra. Then, once we established that $S_{1}$ is irreducible (and it is automatically faithful), we are able to invert the grading of $S$, that is, to replace $S_{i}$ by $S_{-i}$. Then we can assume that $q \leqslant r$. Using interplay between $\oplus_{i \geqslant-1} S_{q i}$ and $\oplus_{i \geqslant-1} S_{(q-1) i}$ we are able to arrive at a contradiction.

The ground field is still assumed to be algebraically closed of characteristic $p>5$.

1. Setup and statements of results. A solvable maximal subalgebra is a maximal subalgebra which is solvable.

1.1. Suppose that $L$ is a simple Lie algebra which contains a solvable maximal subalgebra $H$. Suppose that $L_{0}$ is bad. By Theorem I.5.1 this means, in particular, that $\operatorname{dim} H=1$. Then by Theorem I.2.2, $L=\bigoplus_{i \in \mathbf{Z} / p} L_{i}$ and $L_{i}, i \neq 0$, are irreducible and faithful for $L_{0}$. But then $L_{1} \oplus L_{0}$ is a proper subalgebra (and solvable at that). Thus $L_{0}$ is never bad. Let $A$ be a commutative ideal of $L_{0}$. Then $A$ acts nilpotently on $L_{0}$. By Corollary I.1.4(ii), $A$ acts nilpotently on $G$. This shows that $L_{0}$ gives rise to a long filtration of $L$.

1.2. More generally, consider a Lie algebra $L$ with a maximal subalgebra $L_{0}$ which contains no ideals of $L$. Pick a simple $L_{0}$-submodule $F$ in $L / L_{0}$ and let $L_{-1}$ be its preimage in $L$. Suppose that $\left.\operatorname{Ker} L_{0}\right|_{F} \neq 0$ (which is certainly the case if $L_{0}$ has ideals acting on $L$ nilpotently). Then set $L_{i-1}=\left[L_{i}, L_{i}\right]+L_{i}$ for $i<0$ and $L_{i+1}=$ $\left\{x \in L_{i} \mid\left[x, L_{-1}\right] \subseteq L_{i}\right\}$ for $i \geqslant 0$. In particular, $L_{1}=\left.\operatorname{Ker} L_{0}\right|_{F}$. Then $\left\{L_{i}\right\}$ is a Lie algebra filtration in $L$ (see [12]).

Let $G=\bigoplus_{i \in \mathbf{Z}} G_{i}, G_{i}=L_{i} / L_{i+1}$, be the associated graded Lie algebra. The main result of Part II is

1.3. THEOREM. Suppose that $L_{0}$ is a solvable maximal subalgebra of $L$ and that $L_{0}$ defines a long filtration of $L$. Then there exist a simple Lie algebra $S$ of type $A_{1}$ or $W_{1}$ with standard grading $S=\oplus S_{i}$, a number $m$ and a solvable subalgebra $T$ of derivations of degree 0 of $S \otimes B_{m}$ such that $G \simeq S \otimes B_{m}+T, G_{i}=S_{i} \otimes B_{m}$ for $i \neq 0$, and $G_{0}=S_{0} \otimes B_{m}+T, B_{m}$ has no $T$-invariant ideals. In particular:

(i) $L=L_{-1}$ (i.e., $L_{0}$ is irreducible on $\left.L / L_{0}\right)$;

(ii) $\operatorname{dim}\left(L_{i} / L_{i+1}\right)=m$ if $i \neq 0$ and $L_{i} / L_{i+1} \neq 0$. 
In view of this theorem and M. I. Kuznetsov [9, Theorem 6.1], we have

1.4. Corollary. Suppose that $L$ is simple and contains a solvable maximal subalgebra. Then $L$ is of type $A_{1}$ or $W_{1}$.

1.4.1. REMARK. In characteristic 3, as was already remarked, there are counterexamples to the above statements, see, e.g., M. Frank [4].

1.5. To start proving Theorem 1.3 we denote by $M(G)$ (see $[12, \S 1.5])$ the largest ideal of $G$ contained in $\oplus_{i<0} G_{i}$. We set $\bar{G}=G / M(G), \bar{G}=\oplus \bar{G}_{i}$. Let $A(\bar{G})=$ $\oplus A(\bar{G})_{i}$ (see [12, §1.6]) be the smallest nontrivial ideal of $\bar{G}$. According to [12] there are two cases: degenerate case (when $A(\bar{G})_{1}=0$ ) and nondegenerate case (when $\left.A(\bar{G})_{1} \neq 0\right)$.

1.6. LEMMA. We are in a nondegenerate case. More precisely, if $L_{0}$ is a solvable maximal subalgebra of $L$, then there exists a simple graded Lie algebra $S=\oplus S_{i}$, a natural number $m$ and a solvable subalgebra $T_{0}$ of derivations of degree zero of $S \otimes B_{m}$ such that $A(\bar{G})=S \otimes B_{m}, A(G)_{i}=S_{i} \otimes B_{m}, \bar{G}_{i}=S_{i} \otimes B_{m}$ for $i<0$ and $G_{0}=\bar{G}_{0}=$ $S_{0} \otimes B_{m}+T_{0}$.

Proof. Suppose that $\bar{G}$ is degenerate. Then $\bar{G}_{0}$ contains an ideal of the form $S \otimes B_{u}$ with $S$ simple. This is impossible since $G_{0}$ is a quotient of $L_{0}$, and $L_{0}$ is solvable.

2. The case when $S$ is of the type $A_{1}$ or $W_{1}$. Suppose that $L=\left\{L_{i}\right\}$ is a filtered Lie algebra and $L_{0}$ a solvable maximal subalgebra in $L$ and suppose that $L_{0}$ contains no ideals of $L$. We take notation from the preceding section. As a first step and to simplify reference, we record some properties of $\bar{G}$ in the case when $S$ is type $A_{1}$ or $W_{1}$.

2.1. LemMA. Let $\bar{G}$ be as in $§ 1.5$. Suppose that $S$ is of type $A_{1}$ or $W_{1}$ with standard grading. Write $S_{i}=k e_{i}$. We have:

(i) $\bar{G}_{i}=0$ for $i \leqslant-2 ; \bar{G}_{i}=G_{i}$ for $i \geqslant-1$;

(ii) $G_{i}=A(\bar{G})_{i}$ for $i \neq 0, i \geqslant-1$;

(iii) $G_{i}=e_{i} \otimes B_{m}$ for $i \neq 0, i \geqslant-1$;

(iv) $e_{0} \otimes B_{m} \subseteq G_{0}$ is a commutative ideal of $G_{0}$;

(v) $G_{0}$ acts on $e_{0} \otimes B_{m}$ by derivations of $B_{m} ; B_{m}$ has no $G_{0}$-invariant ideals;

(vi) $e_{0} \otimes 1$ is in the center of $G_{0}$ and it acts as (multiplication by) $i$ on $G_{i}$

(vii) the $G_{i}, p+i, i \geqslant-1$, are irreducible and faithful $G_{0}$-modules;

(viii) $e_{0} \otimes B_{m}$ is the kernel of the action of $G_{0}$ on $G_{p i}, i \neq 0$.

Proof. We have $S_{i}=0$ for $i<-1$, whence (i). Now (ii), (iii), (iv) and (v) follow from M. I. Kuznetsov [9, Theorem 5.1 and Proposition 2.1]. (They can also be derived with little work from results of [12]). Now it is clear from (v) that $e_{0} \otimes 1$ is in the center of $G_{0}$ and that $G_{0}=e_{0} \otimes B_{m}+T_{0}$ with $T_{0} \subseteq 1_{S_{0}} \otimes \operatorname{Der} B_{m}$ acting on $e_{0} \otimes B_{m}$ in such a way that $e_{0} \otimes I$ is not a $T_{0}$-invariant subspace for any ideal $I$ of $B_{m}$. 
Now $e_{0} \otimes 1$ acts as 0 on $G_{0}$ and it acts as $i$ on $G_{i}=A(\bar{G})_{i}, i \geqslant-1$, since $\left[e_{0} \otimes 1, e_{i} \otimes b\right]=\left[e_{0}, e_{i}\right] \otimes b=i e_{i} \otimes b$ for $b \in B_{m}$. Since $G_{i-1}=\left[G_{i}, G_{-1}\right]$ for $i<$ -1 we have that $e_{0} \otimes 1$ acts as $i$ also on $G_{i}, i<-1$. This establishes (vi). To prove (vii) and (viii) note that $e_{0} \otimes b, b \in B_{m}$, acts on $G_{i}=e_{i} \otimes B_{m}, i \geqslant-1, i \neq 0$, as multiplication by $i b$; in particular, it acts trivially on $G_{i}, p \mid i$. So $\operatorname{Ker} G_{0} \mid G_{i} \supseteq e_{0} \otimes B_{m}$ if $i \neq 0, p \mid i$, and the only invariant subspaces for $e_{0} \otimes B_{m}$ on $G_{i}, p+i$, are of the form $e_{i} \otimes I$, where $I$ is an ideal of $B_{m}$. As $T_{0}$ acts faithfully on $B_{m}$ we see that $\operatorname{Ker} G_{0} \mid G_{i}=e_{0} \otimes B_{m}$ if $p \mid i, i \neq 0$, and $\operatorname{Ker} G_{0} \mid G_{i}=0$ if $p+i$. Since $B_{m}$ has no $T_{0}$-invariant ideals it follows that $G_{0}$ acts irreducibly on $G_{i}, p+i$. This concludes the proof of (vii) and (viii).

2.2. RemarK. We assumed that $S$ has the standard grading. The only other possibility is that $S$ has inverse to standard grading; that is, $S_{i}=K e_{-i}$. But then $\left[S_{-1}, S_{-1}\right]=0$; that is, $S$ is type $A_{1}$ and for type $A_{1}$ there is no difference between standard and inverse to standard grading.

2.3. Proposition. Suppose that $S$ is of type $A_{1}$ or $W_{1}$ with standard grading. Then $M(G)=0$.

Proof. Since the grading of $S$ is standard we have $S=\oplus_{i \geqslant-1} S_{i}$. Thus $\bar{G}_{i}=0$ for $i \leqslant-2$ and therefore $M(G)=\bigoplus_{i<-1} G_{i}$. Since $G_{-2} \subseteq M(G)$ we have $\left[G_{-2}, G_{1}\right]=0$ $=\left[G_{-2}, G_{2}\right]$. Therefore $\left[L_{-2}, L_{1}\right] \subseteq L_{0},\left[L_{-2}, L_{2}\right] \subseteq L_{1}$.

Set $M=L, M_{0}=L_{0}, M_{-1}=L_{-2}, M_{i-1}=\left[M_{i}, M_{-1}\right]+M_{i}$ for $i \leqslant-1$, and $M_{i+1}$ $=\left\{x \in M_{i} \mid\left[x, M_{-1}\right] \subseteq M_{i}\right\}$ for $i \geqslant 0$. Then $M_{i}$ is a Lie algebra filtration in $M$ (see proof of Proposition 1.2 in [12]). We know that $\left[M_{-1}, L_{1}\right]=\left[L_{-2}, L_{1}\right] \subseteq L_{0}=M_{0}$. Thus $L_{1} \subseteq M_{1}$. On the other hand, $M_{1}=\left.\left.\operatorname{Ker} M_{0}\right|_{M_{-1} / M_{0}} \subseteq \operatorname{Ker} M_{0}\right|_{L_{-1} / L_{0}}=L_{1}$. Thus $M_{1}=L_{1}$.

Similarly, $\left[M_{-1}, L_{2}\right]=\left[L_{-2}, L_{2}\right] \subseteq L_{1}=M_{1}$ implies that $L_{2} \subseteq M_{2}$. Now $M_{2} / L_{3}$ is a submodule of $G_{1}=L_{1} / L_{2}$. But $G_{1}$ is irreducible (by Lemma 2.1(vii)). Since $M_{2} \neq L_{1}$ we must have $M_{2}=L_{2}$.

Now let $H=\oplus H_{i}, H_{i}=M_{i} / M_{i+1}$, be the graded algebra associated to the filtered algebra $\left\{M_{i}\right\}$. We have $H_{0}=M_{0} / M_{1}=L_{0} / L_{1}=G_{0}, H_{1}=M_{1} / M_{2}=$ $L_{1} / L_{2}=G_{1}$. In particular, $c=e_{0} \otimes 1 \in G_{0}$ (cf. Lemma 2.1) acts as 1 on $H_{1}$ and as 0 on $H_{0}$. Therefore, for $x \in H_{1}$ and $y \in H_{-1}$ we have $[x,[c, y]]=[[x, c] y]+$ $[c[x, y]]=-[x, y]$, whence $\left[H_{1},[c, y]+y\right]=0$. Let $[c, y]+y=v+M_{0}$. Then $\left[M_{1}, v\right] \subseteq M_{1}$, i.e., $\left[L_{1}, v\right] \subseteq L_{1}$. Since $L_{1}$ is not an ideal of $L$ and since $L_{0}$ is maximal in $L$ the inclusion $\left[L_{1}, v\right] \subseteq L_{1}$ implies that $v \in L_{0}=M_{0}$, whence $[c, y]+$ $y=0$, i.e., $c$ acts as -1 on $H_{-1}$. However, $H_{-1}$ involves $G_{-2}$ and $c=e_{0} \otimes 1 \in G_{0}$ acts as -1 on $G_{-1}$ and as -2 on $G_{-2}$. Thus it cannot act as -1 on $H_{-1}$. This contradiction concludes the proof of Proposition 2.3.

2.4. Corollary (M. I. Kuznetsov [9, Theorem 6.1]). If $L$ is simple and $S$ is of type $A_{1}$ or $W_{1}$, then $L$ is of the same type.

3. Initial properties of a minimal counterexample. Our aim is to show that $S$ appearing in Lemma 1.6 is of type $A_{1}$ or $W_{1}$. To show this we pick $S$ of minimal dimension which can appear in Lemma 1.6 and which is not of type $A_{1}$ or $W_{1}$. 
3.1. LeMMA. Let $S$ be as above, $S=\oplus_{i=-q}^{r} S_{i}$. Let $D_{0}=(\operatorname{Der} S)_{0}$. Then:

(i) $S$ is simple;

(ii) $S_{0}$ is solvable;

(iii) $S$ is not of type $A_{1}$ or $W_{1}$ (i.e., $\operatorname{dim} S_{0}>1$ );

(iv) $S_{-1}$ is a faithful $D_{0}$-module;

(v) $S_{-1}$ is an irreducible $D_{0}$-module;

(vi) $S_{i-1}=\left[S_{i}, S_{-1}\right]$ for $i \leqslant-1$;

(vii) for any $x \in S_{i}, x \neq 0, i>0$, we have $\left[x, S_{-1}\right] \neq 0$;

(viii) $S_{-q}$ is irreducible for $S_{0}$;

(ix) $D_{0}$ acts faithfully on every $D_{0}$-submodule of $S_{1}$.

Proof. First, (i) and (ii) hold by construction and (iii) is our assumption. Next, (v), (vi) and (vii) are contained in [12, Theorem 4.1(iii)] and (viii) in [12, Lemma 4.5.1(ii)]. Let us establish (iv). Let $x \in(\operatorname{Der} S)_{0},\left[x, S_{-1}\right]=0$. Then $\left[x, S_{i}\right]=0$ for $i<0$ in view of (vi). Assume by induction that $\left[s, S_{i}\right]=0$ for $i \leqslant m, m \geqslant-1$. Then for $y \in S_{m+1}$ we have $\left[S_{-1}[x, y]\right]=\left[x\left[S_{-1}, y\right]\right] \subseteq\left[x, S_{m}\right]=0$. By (vii) it means that $[x, y]=0$; that is, $\left[x, S_{m+1}\right]=0$. Thus $x$ acts as 0 on $s$, i.e., $x=0$. This proves (iv). Now (ix) follows from [12, Proposition 3.2.1(ii)] and the fact that $S_{0}$ is solvable.

3.2. Definition. A graded Lie algebra $S=\bigoplus_{i=-q}^{r} S_{i}$ of minimal dimension among the Lie algebras satisfying the conclusions of Lemma 3.1 is called a minimal counterexample.

3.3. Lemma. Let $S=\oplus_{i=-q}^{r} S_{i}$ be a minimal counterexample. Let $V \neq 0$ be a $D_{0}$-submodule of $S_{1}$ and let $\tilde{S}=\left\langle S_{-1}, V\right\rangle+D_{0}$. If $\tilde{S} \neq S$, then $\tilde{S} / M(\tilde{S})=R \otimes B_{m}+$ $D_{0}$, where $R=k e_{-1} \oplus k e_{0} \oplus k e_{1}$ is a simple graded Lie algebra of type $A_{1}$. In particular:

(i) $D_{0}$ has a commutative ideal $e_{0} \otimes B_{m}=\left[S_{-1}, V\right]$;

(ii) $D_{0}$ has a nontrivial center, $\operatorname{Center}\left(D_{0}\right) \supset e_{0} \otimes 1$.

Proof. First of all, $\tilde{S} / M(\tilde{S})$ is nondegenerate in view of Lemma 3.1(ix). Thus $A(\tilde{S} / M(\tilde{S}))=R \otimes B_{m}$ (by [12, Theorem 4.1]) with $R$ simple and satisfying conclusions of Lemma 3.1(iv)-(ix). Properties (i) and (ii) of Lemma 3.1 are also satisfied. Since $S$ was a minimal counterexample we have that $R$ is of type $A_{1}$ or $W_{1}$. As $R$ $=\left\langle R_{-1}, R_{1}\right\rangle$ we have that $R$ is of type $A_{1}$. The rest is easily checked.

3.4. Proposition. Let $S$ be a minimal counterexample. Then $S=\left\langle S_{-1}, S_{1}\right\rangle$ (i.e., $S$ is generated by $S_{1}$ and $S_{-1}$ ).

Proof. Suppose not. Let $\tilde{S}=\left\langle S_{-1}, S_{1}\right\rangle+D_{0}$. Then by Lemma 3.3 we have $\tilde{S} / M(\tilde{S})=R \otimes B_{m}+D_{0}$, where $R$ is simple of type $A_{1}, R=\oplus_{i=-1}^{1} R_{i}, \operatorname{dim} R_{i}=1$. We have $S_{-1}=R_{-1} \otimes B_{m}, S_{1}=R_{1} \otimes B_{m}$ and, since $\left[S_{1}, S_{-1}\right]=S_{0}$ (by Lemma III.1.1), we also have $S_{0}=R_{0} \otimes B_{m}$.

Next, since $R_{2}=0$, we have $\left[S_{1}, S_{1}\right]=0$. Both $S_{1}$ and $S_{-1}$ are irreducible for $D_{0}$. If $m=0$ then $\left[S_{-1}, S_{-1}\right]=0$, i.e., $S_{-2}=0$, i.e., $q=1$. But $q>1$ by (an independently proved) Proposition 3.6 below. So $m \geqslant 1$. 
Let $C$ be the maximal ideal of $B_{m}$. Then $R_{0} \otimes C$ acts nilpotently on $S_{-1}$. Therefore (since $S_{j-1}=\left[S_{i}, S_{-1}\right]$ for -1$) R_{0} \otimes C$ acts nilpotently on all $S_{i}, i<0$. By Lemma 3.1(viii) it acts trivially on $S_{-q}$ and therefore $\operatorname{dim} S_{-q}=1$. By Proposition III.1.12(iv), $S_{0}$ acts nontrivially on $S_{-q}$. Therefore $D_{0}$ acting on $B_{m}$ by derivations must preserve $C$. But then it also preserves $R_{-1} \otimes C$, i.e., $D_{0}$ is not irreducible on $S_{-1}$, a contradiction.

3.5. Proposition. Let $S$ be a minimal counterexample. Then $S_{1}$ has at most one proper $D_{0}$-submodule. In particular, $S_{1}$ is indecomposable.

Proof. Suppose the contrary. Let $\tilde{V}, \bar{V}$ be two proper submodules, $\tilde{V} \neq V$. Consider $\tilde{S}=\left\langle S_{-1}, \tilde{V}\right\rangle+D_{0}$ and $\bar{S}=\left\langle S_{-1}, \bar{V}\right\rangle+D_{0}$. By Lemma 3.3 we have $\tilde{S} / M(\tilde{S})=\tilde{R} \otimes B_{\tilde{m}}+D_{0}, \bar{S} / M(\bar{S})=\bar{R} \otimes B_{\bar{m}}+D_{0}$ with $\tilde{R}, \bar{R}$ of type $A_{1}$. In particular, both $\tilde{V}=\tilde{R}_{1} \otimes B_{\tilde{m}}$ and $\bar{V}=\bar{R}_{1} \otimes B_{\bar{m}}$ are irreducible for $D_{0}$.

We claim that $\tilde{V}+\bar{V}=S_{1}$. If not, then $\tilde{V}+\bar{V}$ is a proper submodule of $S_{1}$, and we can assume that it is one of our submodules $\tilde{V}$ or $\bar{V}$, say $\bar{V}$. Then $\bar{V} \supseteq \tilde{V}$. Since both $\bar{V}$ and $\bar{V}$ are irreducible we must have $\bar{V}=\bar{V}$, a contradiction.

Thus $\bar{V}+\tilde{V}=S_{1}$. Now we know that $S_{-2} \subseteq M(\tilde{S})$ and $S_{-2} \subseteq M(\bar{S})$. Therefore $\left[S_{-2}, \tilde{V}\right]=0,\left[S_{-2}, \bar{V}\right]=0$, whence $\left[S_{-2}, S_{1}\right]=0$. Since $\oplus_{i>0} S_{i}=\left\langle S_{1}\right\rangle$ we have that $S_{-2} \subseteq M(S)$, and since $S$ is simple, $S_{-2}=0$. Thus $q=1$, which is a contradiction in view of the following

\subsection{Proposition. Let $S$ be a minimal counterexample. Then $q \geqslant 2$.}

Proof. Suppose $q=1$. As $S$ is simple it follows that $S_{-1}$ is irreducible for $S_{0}$ (Proposition III.1.12(iii) with $q=1$ ). Now our claim follows by M. I. Kuznetsov [9, Theorem 5.1].

\section{Proof that $S_{1}$ is irreducible for $D_{0}$.}

4.1. Proposition. Let $S=\bigoplus_{i=-q}^{r} S_{i}$ be a minimal counterexample. Then $S_{1}$ is irreducible for $D_{0}$.

To start the proof we suppose the contrary. Let $V$ be a proper submodule of $S_{1}$. Set $\tilde{S}_{i}=S_{i}$ for $i<0, \tilde{S}_{0}=D_{0}$ and $\tilde{S}_{1}=V, \tilde{S}_{i+1}=\left\{x \in S_{i+1} \mid\left[S_{-1}, x\right] \subseteq \tilde{S}_{i}\right\}$ for $i \geqslant 1$. Set $\tilde{S}=\oplus \tilde{S}_{i}$. It is a subalgebra by Lemma III.1.8.

4.2. LeMMA. (i) $S_{1} / V$ is irreducible;

(ii) $\tilde{S}$ is a maximal subalgebra of $S+D_{0}$;

(iii) $\tilde{S} \cap S_{1}=V$;

(iv) $S_{1} / V$ is contained in every nontrivial $\tilde{S}$-submodule of $\left(S+D_{0}\right) / \tilde{S}$;

(v) $\tilde{S} / M(\tilde{S})=R \otimes B_{m}+D_{0}$, where $R$ is a simple Lie algebra of type $A_{1}$ or $W_{1}$ with standard grading.

Proof. First, (i) follows from Proposition 3.5. Then (ii) and (iv) follow from Proposition III.1.9. Next, (iii) is just the definition of $\tilde{S}_{1}$. 
To prove (v) note that $\tilde{S} / M(\tilde{S})$ is nondegenerate in view of Lemma 3.1(ix). Thus $A(\tilde{S} / M(\tilde{S}))=R \otimes B_{m}$ (by [12, Theorem 4.1]) with $R$ simple and satisfying conclusions of Lemma 3.1(iv)-(ix). Properties (i) and (ii) of Lemma 3.1 are also satisfied. Since $S$ is a minimal counterexample we have that $R$ is of type $A_{1}$ or $W_{1}$ as required.

4.3. Corollary. (i) $M(\tilde{S})=\oplus_{i \leqslant-2} S_{t} \neq 0$.

(ii) Write $R=\bigoplus_{i \geqslant-1} k e_{i}$. Then $e_{0} \otimes 1$ belongs to the center of $D_{0}$ and acts as multiplication by $i$ on each $S_{i}$.

Proof. By Lemma $4.2(\mathrm{v})$ we know that $(\tilde{S} / M(\tilde{S}))_{-2}=0$. So $S_{-2} \subseteq M(\tilde{S})$. Therefore $M(\tilde{S})=\oplus_{i \leqslant-2} S_{i}$. Since $q>1$ we have $M(\tilde{S}) \neq 0$. This proves (i).

We have $\left[e_{0} \otimes 1, e_{-1} \otimes b\right]=\left[e_{0}, e_{-1}\right] \otimes b=-e_{-1} \otimes b$ for $b \in B_{m}$. Therefore $e_{0} \otimes$ 1 acts as -1 on $S_{-1}=\tilde{S}_{-1}$. Since $D_{0}$ is faithful on $S_{-1}$ it follows that $e_{0} \otimes 1$ is in the center of $D_{0}$. Since $S_{-1}=\left[S_{i}, S_{-1}\right]$ for $i<0$, it follows that $e_{0} \otimes 1$ acts as $i$ on $S_{i}$, $i<0$. Suppose that $e_{0} \otimes 1$ acts as $i$ on all $S_{i}, i \leqslant t$. Then for $x \in S_{-1}, y \in S_{t+1}$ we have

$$
\begin{aligned}
{\left[x\left[e_{0} \otimes 1, y\right]\right] } & =\left[\left[x, e_{0} \otimes 1\right], y\right]+\left[e_{0} \otimes 1,[x, y]\right] \\
& =[x, y]+t[x, y]=(t+1)[x, y] .
\end{aligned}
$$

In view of Lemma 3.1 (vii) this implies that $e_{0} \otimes 1$ acts as $(t+1)$ on $S_{t+1}$. This proves (ii).

4.4. Now set $L=S+D_{0}, L_{0}=\tilde{S}$, and let $L_{-1}$ be a minimal subspace of $L$ such that $L_{-1} \supset L_{0}, L_{-1} \neq L_{0}$ and $\left[L_{-1}, L_{0}\right] \subseteq L_{-1}$. Let $\left\{L_{i}\right\}$ be the resulting filtration (cf. §1.2).

LEMMA (i) $L_{-1}$ is the $L_{0}$-submodule of $L$ generated by $S_{1}+L_{0}$;

(ii) $L_{1}=M(\tilde{S}) \neq 0$; in particular, the filtration is long.

Proof. The first claim directly follows from Lemma 4.2(iv). To prove the second claim, note that $M(\tilde{S})$ is a nilpotent ideal of $L_{0}$, and $M(\tilde{S})$ acts nilpotently on $L$. Therefore, $M(\tilde{S})$ acts trivially on the irreducible $L_{0}$-module $L_{-1} / L_{0}$. Thus $M(\tilde{S}) \subseteq$ $L_{1}$. Next, $L_{1}$ is a nilpotent ideal of $L_{0}$, and the algebra $\tilde{S} / M(\tilde{S})$ does not have nilpotent ideals (by Lemma 4.2(v)). So $L_{1}=M(\tilde{S})$.

4.5. LemMA. Let $F=\oplus F_{i}$ be the graded Lie algebra associated to the filtered Lie algebra $\left\{L_{i}\right\}$ :

(i) $F / M(F)$ is nondegenerate;

(ii) $F_{0}=R \otimes B_{m}+D_{0}$;

(iii) $\left[F_{1}, F_{-1}\right]=R \otimes B_{m}$;

(iv) $F_{-1} \supset S_{1} / V$;

(v) $A(F / M(F))_{1} \supseteq S_{-2}$.

(Inclusions and equalities here and below have the natural interpretation.)

Proof. We clearly have (iv) by Lemma 4.4(i), and (ii) by Lemma 4.4(ii). In particular, $S_{-1} \subseteq F_{0}$. By construction of the graded Lie algebra we see that [ $\left.S_{-1}, S_{1} / V\right]=0$ in $F$. Since $F_{-1}$ is generated by $S_{1} / V$ as an $F_{0}$-module by Lemma 
4.4(i), to show that $\left[F_{1}, F_{-1}\right] \subseteq R \otimes B_{m}$ it is sufficient to check that $\left[F_{1}, S_{1} / V\right] \subseteq R$ $\otimes B_{m}$. But $\left[\sum_{i \leqslant-2} S_{i}, S_{1} / V\right] \subseteq \sum_{i \leqslant-1} S_{i}$. So $\left[F_{1}, S_{1} / V\right] \cap F_{0}=S_{-1} \subseteq R \otimes B_{m}$. So $\left[F_{1}, F_{-1}\right] \subseteq R \otimes B_{m}$ and $\left[F_{1}, F_{-1}\right] \supseteq\left[S_{-2}, S_{1} / V\right]=S_{-1}$. Since $\left[F_{1}, F_{-1}\right]$ is an ideal of $F_{0}$ we must have $\left[F_{1}, F_{-1}\right]=R \otimes B_{m}$. This proves (iii).

To prove (v) we have to establish first that $F_{1} \supseteq S_{-2}$, i.e. that $S_{-2} \cap L_{2}=0$. For $x \in S_{-2} \cap L_{2}$ we have $\left[x, L_{-1}\right] \subset L_{1}=M(\tilde{S})$. Since, $S_{1} \subset \mathrm{L}_{-1}$ this implies that [ $\left.x, S_{1}\right]=0$ whence, by Proposition 3.4, $x \in M(S)=0$. Now by (iii) (and in view of Corollary 4.3(ii)) we have that $c=e_{0} \otimes 1 \in A(F / M(F))_{0}$. Since $c$ acts as -2 on $S_{-2}$ it follows that $S_{-2}=\left[c, S_{-2}\right] \subseteq A(F / M(F))$. This proves (v). Since it is now established that $A(F / M(F))_{1} \neq$ we also have (i).

4.6. Lemma. $A(F / M(F))=P \otimes B_{m}$, where $P$ is a simple graded Lie algebra, $P=\bigoplus_{i=-s}^{\prime} P_{i}$.

Proof. We have $A(F / M(F))=P \otimes B_{d}$ with $P$ simple graded, $P=\bigoplus_{i=-s}^{\prime} P_{i}$ in view of Lemma 4.5(i) and [12, Theorem 4.1]. By Lemma 4.5(iii) we have $P_{0}=R \otimes$ $B_{m-d}$. By [12, Lemma 4.5.1(ii)] we have that $P_{-s}$ is irreducible for $P_{0}$, and by Proposition III.1.12(iii) we see that $\left[P_{0}, P_{-s}\right] \neq 0$. Let $C$ be the maximal ideal of $B_{m-d}$. Then the preimage of $R \otimes C B_{m}$ in $L$ acts nilpotently on $S$. Therefore $R \otimes C B_{m}$ acts nilpotently on $\oplus_{i \geqslant 0} F_{i}$, i.e., $R \otimes C$ acts nilpotently on $\oplus_{i \geqslant 0} P_{i}$. It follows that $R \otimes C$ acts nilpotently on $P$ (if $n \in R \otimes C$ has an eigenvalue $\lambda \neq 0$ on some $P_{j}, j<0$, then using commutation with $\bigoplus_{i>0} P_{i}$ we shall see that $n$ has the same eigenvalue on some $\left.P_{r}, r \geqslant 0\right)$. Since $P_{-s}$ is irreducible, $R \otimes c$ acts trivially on $P_{-s}$. Since $\left[P_{0}, P_{-s}\right] \neq 0$ this means that $D_{0}$ preserves the ideal $R \otimes C B_{m}$ of $R \otimes B_{m}$. But then $D_{0}$ does not act irreducibly on $S_{-1}$ (and neither does $F_{0}$ act irreducibly on $\left.F_{-1}\right)$. Thus $m-d=0$, i.e., $d=m$. This proves our claim.

4.7. Let $Q$ be the maximal ideal of $A(F / M(F))$. Then $P=A(F / M(F)) / Q$. Let $\mathscr{T}$ be the one-dimensional subtorus of Aut $S$ defining the grading $S=\oplus S_{i}$ (see [12, $\S 14]$ ). Since $L_{0}=\tilde{S}, L_{1}=M(\tilde{S})$, and $L_{-1}$ (by Lemma $4.4(\mathrm{i})$ ) are invariant under $\mathscr{T}, \mathscr{T}$ goes over to $F$. Next, $Q$ is also invariant under $\mathscr{T}$. So we consider $\mathscr{T}$ as acting also on $P$ and preserving each $P_{i}$. If $\chi$ is the character of $\mathscr{T}$ on $S_{1}$ we can write $P_{i j}=\left\{x \in P_{i} \mid t \cdot x=j \chi(t) \cdot x\right\}$. This gives us a bigrading of $P$.

LemMa. (i) $\left[P_{i j}, P_{k l}\right]=P_{i+k, j+l}$;

(ii) $P_{0}=\bigoplus_{i \geqslant-1} P_{0, i}$, and $\operatorname{dim} P_{0, i}=1$ if $P_{0, i} \neq 0$;

(iii) $P_{-1}=\oplus_{i \geqslant 1} P_{-1, i}$;

(iv) $P_{1}=\bigoplus_{i \leqslant-2} P_{1, i}$.

Proof. We have: (i) is true since $P_{i j}$ is a bigrading; (ii) is true by Corollary 4.3(ii); (iii) is true since $F_{-1}$ is generated by $S_{1} / V$ and $\left[S_{1} / V, S_{-1}\right]=0$; (iv) is true because $L_{1} \subseteq \bigoplus_{i \leqslant-2} S_{i}$.

4.8. Lemma. (i) There exist $e_{0.0} \in P_{0,0}, e_{0.1} \in P_{0.1}$ and $e_{0,-1} \in P_{0 .-1}$ such that $\left[e_{0.1}, e_{0,-1}\right]=-e_{00}$ and $e_{00}$ acts as (a multiplication by) $j$ on every $P_{i j}$;

(ii) there exist $e_{-1.1} \in P_{-1.1}$ and $e_{1,-2} \in P_{1,-2}$ such that $\left[e_{-1.1}, e_{1,-2}\right]=e_{0 .-1}$.

Proof. We have $P_{0}=R$ so $P_{0}=\oplus_{i \geqslant-1} k e_{i}$. Set $e_{0.0}=e_{0}, e_{0.1}=e_{1}$ and $e_{0 .-1}=e_{-1}$. Then (i) holds in view of Corollary 4.3 (ii). 
To prove (ii) recall that $\left[S_{-2}, S_{1} / V\right]=S_{-1}$, where $S_{-2} \subseteq F_{1}, S_{1} / V \subseteq F_{-1}$ and $S_{-1} \subseteq\left[F_{1}, F_{-1}\right]$. This gives us (ii).

4.9. Set $e_{1,-i-1}=\left(\operatorname{ad} e_{0,-1}\right)^{i-1} e_{1,-2} \in P_{1,-i-1}$ for $i \geqslant 1$ and $e_{-1, i+1}=\left(\operatorname{ad} e_{0,1}\right)^{i} e_{-1,1}$. We are going to compute certain commutators. First of all, in view of Lemma 4.8(i), (ii) we have

$$
\begin{gathered}
{\left[e_{0,1}, e_{0,-1}\right]=-e_{0,0},} \\
{\left[e_{0,0}, e_{i j}\right]=j e_{i, j},} \\
{\left[e_{-1,1}, e_{1,-2}\right]=e_{0,-1}}
\end{gathered}
$$

Next, we have by Lemma 4.7(iii), (iv)

$$
\begin{aligned}
& {\left[e_{0,1}, e_{1,-2}\right]=0,} \\
& {\left[e_{0,-1}, e_{-1,1}\right]=0}
\end{aligned}
$$

Now we claim that

$$
\left[e_{0,1}, e_{1,-i}\right]=(i(i-1) / 2-1) e_{1,-i+1} \text { for } i \geqslant 2 \text {. }
$$

We prove it by induction. It holds if $i=2$ in view of (4.9.4). Suppose it holds for $i \leqslant m$. Then

$$
\begin{aligned}
{\left[e_{0,1}, e_{1,-m-1}\right] } & =\left[e_{0,1}\left[e_{0,-1}, e_{1,-m}\right]\right]=\left[\left[e_{0,1}, e_{0,-1}\right] e_{1,-m}\right]+\left[e_{0,-1}\left[e_{0,1}, e_{1,-m}\right]\right] \\
& =\left[-e_{0,0}, e_{1,-m}\right]+\left[e_{0,-1},(m(m-1) / 2-1) e_{1,-m+1}\right] \\
& =m e_{1,-m}+(m(m-1) / 2-1) e_{1,-m}=((m+1) m / 2-1) e_{1,-m},
\end{aligned}
$$

as required.

Next, since $\left[e_{-1,1}, e_{1,-i}\right],\left[e_{-1,2}, e_{1,-i-1}\right] \in P_{0,-i+1}$ and $P_{0,-i+1}=0$ for $i>2$ we have

$$
\begin{array}{ll}
{\left[e_{-1,1}, e_{1,-i}\right]=0} & \text { for } i>2, \\
{\left[e_{-1,2}, e_{1,-i}\right]=0} & \text { for } i>3 .
\end{array}
$$

Next we have $\left[e_{-1,2}, e_{1,-2}\right]=\left[\left[e_{0,1}, e_{-1,1}\right] e_{1,-2}\right]=\left[e_{0,1}\left[e_{-1,1}, e_{1,-2}\right]\right]=\left[e_{0,1}, e_{0,-1}\right]=$ $-e_{00}$; that is,

$$
\left[e_{-1,2}, e_{1,-2}\right]=-e_{00} \text {. }
$$

Now $\left[\left[e_{0,-1}, e_{0,1}\right] e_{-1,1}\right]=\left[e_{0,0}, e_{-1,1}\right]=e_{-1,1}$; that is, by (4.9.5)

$$
\left[e_{0,-1}, e_{-1,2}\right]=e_{-1,1} \text {. }
$$

Next $\left[e_{-1,2}, e_{1,-3}\right]=\left[\left[e_{0,1}, e_{-1,1}\right] e_{1,-3}\right]=\left[\left[e_{0,1}, e_{1,-3}\right] e_{-1,1}\right]=\left[2 e_{1,-2}, e_{-1,1}\right]=-2 e_{0,-1}$ (we used the definition of $e_{-1,2},(4.9 .7),(4.9 .6)$ with $i=3$, and (4.9.3)). Thus

$$
\left[e_{-1,2}, e_{1,-3}\right]=-2 e_{0,-1} \text {. }
$$

Now

$$
\begin{aligned}
{\left[e_{1,-3}, e_{-1,3}\right] } & =\left[e_{1,-3},\left[e_{0,1}, e_{-1,2}\right]\right]=\left[\left[e_{1,-3}, e_{0,1}\right] e_{-1,2}\right]+\left[e_{0,1}\left[e_{1,-3}, e_{-1,2}\right]\right] \\
& =\left[-2 e_{1,-2}, e_{-1,2}\right]+\left[e_{0,1}, 2 e_{0,-1}\right]=-2 e_{00}-2 e_{00}=-4 e_{00} .
\end{aligned}
$$

We used the definition of $e_{-1.3}$, the Jacobi identity (4.9.6) with $i=3$, (4.9.11), (4.9.9) and (4.9.1). Thus

$$
\left[e_{1,-3}, e_{-1,3}\right]=-4 e_{0,0}
$$


Next, $\left[e_{1,-2}, e_{-1,4}\right]=\left[e_{1,-2}\left[e_{0,1}, e_{-1,3}\right]\right]=\left[e_{0,1}\left[e_{1,-2}, e_{-1,3}\right]\right]=\left[e_{0,1}, \lambda e_{0,1}\right]=0$. We used the definition of $e_{-1,4},(4.9 .4)$ and Lemma 4.7(i), (ii). Thus

$$
\left[e_{1,-2}, e_{-1,4}\right]=0 \text {. }
$$

Now $\left[e_{1,-4}, e_{-1,3}\right]=\left[e_{1,-4},\left[e_{0,1}, e_{-1,2}\right]\right]=\left[\left[e_{1,-4}, e_{0,1}\right] e_{-1,2}\right]=\left[-5 e_{1,-3}, e_{-1,2}\right]=$ $-10 e_{0,-1}$. We used the definition of $e_{-1,3},(4.9 .8)$ with $i=4,(4.9 .6)$ with $i=4$ and, finally, (4.9.11). We got

$$
\left[e_{1,-4}, e_{-1,3}\right]=-10 e_{0,-1} \text {. }
$$

Finally,

$$
\begin{aligned}
{\left[e_{1,-4}, e_{-1,4}\right] } & =\left[e_{1,-4}\left[e_{0,1}, e_{-1,3}\right]\right]=\left[\left[e_{1,-4}, e_{0,1}\right] e_{-1,3}\right]+\left[e_{0,1}\left[e_{1,-4}, e_{-1,3}\right]\right] \\
& =\left[15 e_{1,-3}, e_{-1,3}\right]+\left[e_{0,1},-10 e_{0,-1}\right]=20 e_{0,0}+10 e_{0,0}=30 e_{0,0}
\end{aligned}
$$

We used the definition of $e_{-1,4}$, the Jacobi identity, (4.9.6) with $i=4$, (4.9.14), (4.9.12) and (4.9.1). Our result is

$$
\left[e_{1,-4}, e_{-1,4}\right]=30 e_{00} .
$$

4.10. Now look at the subalgebra of $P$ generated by

$$
E_{1}=e_{-1,2}, \quad E_{2}=e_{-1,4}, \quad H=e_{0,0}, \quad F_{1}=e_{1,-2}, \quad F_{2}=e_{1,-4} .
$$

We have $\left[E_{i}, F_{j}\right]=\lambda_{i} \delta_{i j} H, \lambda_{1}=-1, \lambda_{2}=-30$, and $\left[H, E_{i}\right]=a_{i} E_{i}, a_{i} \neq 0$, which makes our algebra into a contragredient algebra. As $p>5$ we have $\lambda_{1} \neq 0, \lambda_{2} \neq 0$. By V. Kac [5, Lemma 2.1 or 2.3] it follows that our subalgebra and, therefore, $P$, and, therefore, $S$ are infinite-dimensional. This contradiction establishes that $S_{1}$ must be irreducible.

5. Proof of Theorem 1.3 concluded. We have established in the previous section that $S_{1}$ is irreducible. It is faithful by Lemma 3.1(ix). This makes it possible to invert the grading of $S=\oplus_{i=-q}^{r}$ without violating conclusions of Lemma 3.1. So we can (and shall) assume that $q \leqslant r$. For easy reference we record

5.1. LEMMA. (i) $r \geqslant((p-1) / 2) q ; q>1$;

(ii) $S_{1}$ is irreducible;

(iii) $S$ is generated by $S_{1}$ and $S_{-1}$;

(iv) $S_{0} \subseteq\left[S_{-q}, S_{q}\right]+\left[S_{-q+1}, V\right]$ for any $D_{0}$-submodule $V \neq 0$, of $S_{q-1}$.

Proof. First, (i) follows from Theorem III.2.1 (applicable in view of Remark III.2.1.1) and Proposition 3.6. Now (ii) and (iii) are Propositions 4.1 and 3.4, respectively. Finally, (iv) is Proposition III.1.7(ii).

5.2. LEMMA. Let $V \neq 0$ be a $D_{0}$-submodule of $S_{i}$.

(i) If $p+i$, then $S_{0}$ acts faithfully on $V$.

(ii) If $p \mid i$, then every commutative ideal of $D_{0}$ (in particular, the last nonzero term of the derived series of $S_{0}$ ) acts nilpotently on $V$ (and therefore trivially if $V$ is irreducible).

Proof. Let $I$ be a commutative ideal of $D_{0}$. Then $I$ is a nilpotent ideal, and (ii) follows from Corollary III.1.11(ii). If $R$ is the kernel of $S_{0}$ on $V$, then $R$ is an ideal of $D_{0}$. Let $J$ be the last nonzero term of the derived series of $R$. Then $J$ is a nilpotent ideal of $D_{0}$ and (i) follows from Corollary III.1.11(i). 
5.3. Proposition. Suppose $p+q-1, q\rangle 2$. Then $\left\langle S_{-q+1}, S_{q-1}\right\rangle$ is isomorphic (as a graded Lie algebra) to $R \otimes B_{n}$, where $\tilde{R}$ is graded of type $A_{1}$. In particular, $S_{-q+1}$ and $S_{q-1}$ are irreducible.

Proof. Let $V$ be a $D_{0}$-irreducible submodule of $S_{-q+1}$. Consider $\tilde{S}=\left\langle V, S_{q-1}\right\rangle$ $+D_{0}$. By Proposition III.1.5(iv) (applicable in view of Proposition III.1.4 and the Remark following it) we have $[V, x] \neq 0$ for any $x \in S_{q-1}, x \neq 0$. By Lemma 5.2, $S_{0}$ is faithful on $V$. Consider $\bar{D}_{0}=\left\{x \in D_{0} \mid[x, V]=0\right\}$. Then $\bar{D}_{0}$ is an ideal of $D_{0}$ and $\left[\bar{D}_{0}, S_{0}\right]=0$ as both are ideals and $S_{0}$ is faithful on $V$. Then $\left[\bar{D}_{0}, S_{q-1}\right]=0$ as well (for if $\left[\bar{D}_{0}, x\right] \neq 0$ for $x \in S_{q-1}$, then $0 \neq\left[V\left[\bar{D}_{0}, x\right]\right]=\left[\bar{D}_{0}[V, x]\right] \subseteq\left[\bar{D}_{0}, s_{0}\right]=0$, a contradiction). Thus $\bar{D}_{0}$ is an ideal of $\tilde{S}$. Replacing $\tilde{S}$ by $\bar{S}=\tilde{S} / \bar{D}_{0}$ we see that $\bar{S}$ satisfies properties (i), (ii), (iv), (v), (vi), (viii) and (ix) of Lemma 3.1. Consider the ideal $N(\bar{S})$ constructed in the proof of [12, Lemma 4.5.1]: $N^{0}(\bar{S})=0, N^{1+1}(\bar{S})=x$ $\left.\in \bigoplus_{j \geqslant 0} \bar{S}_{j(q-1)} \mid\left[S_{-q+1}, x\right] \subseteq N^{\prime}(\bar{S})\right\}, N(\bar{S})=\bigcup N^{i}(\bar{S})$. Then $N(\bar{S}) \cap S_{q-1}=0($ since $[V, x] \neq 0$ for $\left.x \neq 0, x \in S_{q-1}\right)$. Then $\bar{S} / N(\bar{S})$ satisfies all conclusions of Lemma 3.1 , except (iii). Therefore, since $S$ is a minimal counterexample, $\bar{S}=\bar{R} \otimes B_{m}+$ $D_{0} / \bar{D}_{0}$ with $\bar{R}$ of type $A_{1}$ or $W_{1}$. Since $\bar{R}$ is generated by $S_{-q+1}$ and $S_{q-1}$ we see that $\bar{R}$ must be of type $A_{1}$. In particular, $S_{q-1}$ is irreducible.

Now consider $H=\left\langle S_{-q-1}, S_{q-1}\right\rangle$. Again $S_{0}$ is faithful on $S_{q-1}$ by the preceding part and $\left[x, S_{q-1}\right] \neq 0$ for $x \neq 0, x \in S_{-q+1}$ (by Propositions III.1.7(i) and III.1.12(vi)). Then $H / M(H)$ satisfies all conclusions of Lemma 3.1 except possbily (iii). As $S$ is a minimal counterexample, and $q>2$ implies that $\operatorname{dim} H<\operatorname{dim} S$, we see that $H / M(H)=\tilde{R} \otimes B_{m}+D_{0}$ with $\tilde{R}$ of type $A_{1}$ or $W_{1}$. Again, since $\tilde{R}$ is generated by $S_{-q+1}$ and $S_{q-1}$ we must have that $\tilde{R}$ is of type $A_{1}$. And then $S_{-q+1}$ must be irreducible. This concludes the proof.

\subsection{Corollary. $p+q$.}

Proof. If $p \mid q-1$, then there is a nothing to prove. So assume that $p+q-1$, but $p \mid q$. Then $\left[S_{-q+1}, S_{q-1}\right]$ is (by Proposition 5.3) a nilpotent ideal of $D_{0}$. Since $S_{-q}$ is irreducible it follows from Lemma 5.2(ii) that $\left[S_{-q+1}, S_{q-1}\right]$ acts trivially on $S_{-q}$. But this contradicts Proposition III.1.5(ii).

5.5. Since $p+q$ we have that $S_{0}$ is faithful and irreducible on $S_{-q}$ (by Lemma 5.2(i)). By Proposition III.1.12 the algebra $\oplus_{i \geqslant-1} S_{i q}$ satisfies the conditions of M. I. Kuznetsov [9, Theorem 5.1]. Therefore, $\oplus_{i \geqslant-1} S_{i q}=R \otimes B_{m}+S_{0}$ with $R$ simple graded of type $A_{1}$ or $W_{1}$ with standard grading (magnified $q$ times). We write $R_{i q}=k e_{i q}$ so that $S_{i q}=e_{i q} \otimes B_{m}$. We write $e_{i q}$ for $e_{i q} \otimes 1$.

Proposition. (i) $S_{i q}=e_{i q} \otimes B_{m}, i \neq 0$, are irreducible for $S_{0}$;

(ii) $e_{0} \otimes B_{m}$ is a commutative ideal of $D_{0}$;

(iii) $e_{0}$ is in the center of $D_{0}$;

(iv) $e_{0}$ acts as $i / q$ on $S_{i}$.

The statements (i)-(iii) are, essentially, proved in the proof of Lemma 2.1. To prove (iv) note that $e_{0}$ acts as -1 on $S_{-q}$. Since $e_{0}$ is in the center of $D_{0}$ it acts as a 
scalar, say $\lambda$, on $S_{-1}$. Therefore it acts as $\lambda i$ on $S_{i}$. In particular, it acts as $-\lambda q=-1$ on $S_{-q}$. Thus $\lambda=1 / q$, whence (iv).

5.6. Proposition. (i) ad $e_{-q}: S_{i q+j} \rightarrow S_{i q+j-q}$ is injective for $i \geqslant 0, q>j>0$;

(ii) $\operatorname{ad} e_{-q} \circ$ ad $e_{q}=-(q-j) q^{-1} \operatorname{Id}_{S_{-u+1}}$ for $0<j<q$.

(iii) ad $e_{q}: S_{-q+j} \rightarrow S_{j}$ and ad $e_{-q}: S_{j} \rightarrow S_{-q+j}, 0<j<q$, are bijective if $p+(q-$ $j)$; ad $e_{q}: S_{-q+j} \rightarrow S_{j}$ is trivial if $p+(q-j)$.

(iv) ad $e_{q} \circ$ ad $e_{-q}=(q-j) q^{-1} \operatorname{Id}_{S,}$ if $0<j<q$.

Proof. Set $\tilde{S}_{0}=\left[S_{-q}, S_{q}\right]$ and $\tilde{S}_{i}=S_{i}$ for $i \neq 0$. We know that ad $e_{-q}: \tilde{S}_{i q} \rightarrow \tilde{S}_{(i-1) q}$ is an isomorphism of vector spaces for $i \geqslant 0$ (as $\left[e_{-q}, e_{q i} \otimes b\right]=\left[e_{-q}, e_{q i}\right] \otimes b=$ $\left.e_{q(i-1)} \otimes b\right)$. Now take $x \in \tilde{S}_{i q+j}, i \geqslant 0, q>j>0$, and assume $\left[e_{-q}, x\right]=0$. Then $0=\left(\operatorname{ad} S_{-1}\right)^{j}\left[e_{-q}, x\right]=\left[e_{-q},\left(\operatorname{ad} S_{-1}\right)^{j} x\right]$. But $\left(\operatorname{ad} S_{-1}\right)^{j} x \neq 0$ if $x \neq 0$ and ad $e_{-q}$ is injective on $\tilde{S}_{i q}, i \geqslant 0$. Therfore $x=0$. This proves (i).

Now for $x \in S_{-q+j}, 0<j<q$, we have $\left[e_{-q}\left[e_{q}, x\right]\right]=\left[\left[e_{-q}, e_{q}\right] x\right]=\left[\varepsilon_{v}, x\right]=$ $-(q-j) q^{-1} x$, whence (ii). If $p+(q-j)$, then since ad $e_{-q} \circ \operatorname{ad} e_{q}(x)=-(q-j) q^{-1} x$ with $(q-j) q^{-1} \neq 0$ we must have that ad $e_{q}(x) \neq 0$; that is, ad $e_{q}$ is injective. Thus $\operatorname{dim} S_{j} \geqslant \operatorname{dim} S_{-q+j}$. But ad $e_{-q}: S_{j} \rightarrow S_{-q+j}$ is injective as well (by (i)), so $\operatorname{dim} S_{j}=$ $\operatorname{dim} S_{-q+j}$ and both ad $e_{q}: S_{-q+j} \rightarrow S_{j}$ and ad $e_{-q}: S_{j} \rightarrow S_{-q+j}$ are bijective. If $p \mid q-j$ and ad $e_{q}(x) \neq 0$ for $x \in S_{q+j}$, then also ad $e_{-q}\left(\operatorname{ad} e_{q}(x)\right) \neq 0$ (by (i)). But ad $e_{-q} \circ$ ad $e_{q}=0_{S_{-q+}}$ if $p \mid(q-j)$. This concludes the proof of (iii).

To prove (iv) first assume that $p \mid q-j$. Then ad $e_{q}: S_{-q+j} \rightarrow S_{j}$ is trivial so (iv) holds. So $p+q-j$. Take a basis in $S_{j}$ and a basis in $S_{-q+j}$. Then ad $e_{q}: S_{-q+j} \rightarrow S_{j}$ is given by a matrix, say $A$, and ad $e_{-q} S_{j} \rightarrow S_{-q+j}$ is given by a matrix, say $B$. By (ii) we know that $B A=-(q-j) \cdot q^{-1}$. Id. By (iii) both $B$ and $A$ are square matrices. Therefore they are invertible and $B A=A B=-(q-1) q^{-1}$ Id, which proves (iv).

\subsection{Proposition. $q>2$.}

Proof. Suppose $q=2$. Take $x \in S_{i}$ and apply Proposition 5.6(iv) with $j=1$. Then $q-j=1$ so

$$
\begin{aligned}
-\frac{1}{2} x & =\left[e_{2}\left[e_{-2}, x\right]\right]=\left[\left[e_{2}, e_{-2}\right] x\right]+\left[e_{-2}\left[e_{2}, x\right]\right] \\
& =-\left[e_{0}, x\right]+\left[e_{-2}\left[e_{2}, x\right]\right]=-\frac{1}{2} x+\left[e_{-2}\left[e_{2}, x\right]\right] .
\end{aligned}
$$

So $\left[e_{-2}\left[e_{2}, x\right]\right]=0$. Now apply Proposition 5.6(i) with $i=1, j=1$. This gives $\left[e_{2}, x\right]=0$. Therefore $\left[e_{2}, S_{1}\right]=0$. Now take $e_{2} \otimes b \in S_{2}$ and write $e_{2} \otimes b=\left[e_{0} \otimes\right.$ $\left.b, e_{2}\right]$. Then $\left[e_{2} \otimes b, S_{1}\right]=\left[\left[e_{0} \otimes b, e_{2}\right] S_{1}\right]=\left[\left[e_{0} \otimes b, S_{1}\right] e_{2}\right] \subseteq\left[S_{1}, e_{2}\right]=0$. Thus $\left[S_{2}, S_{1}\right]=0$.

Now $S=\left\langle S_{1}, S_{-1}\right\rangle$ by Lemma 5.1 (iii). So $\left[S_{2}, S_{1}\right]=0$ means that $S_{3}=0$, that is $S=\oplus_{i=-2}^{2} S_{i}$. Now Theorem III.2.1 implies that $S$ is classical, whence a contradiction, since there are no classical graded Lie algebras with $q=2$ and $S_{0}$ solvable.

5.7.1. REMARK. It is curious that an algebra with similar properties has appeared in [14, Theorem 2.11].

\subsection{Proposition. $p \mid q-1$.}


Proof. Suppose $p+q-1$. Then by Proposition 5.3 (which is applicable because of Proposition 5.7) we have that $\left[S_{-q+1}, S_{q-1}\right]=\tilde{R} \otimes B_{n}$ is a commutative ideal $I_{q-1}$ in $S_{0}$. In all cases, by Proposition 5.5(ii) we see that $\left[S_{-q}, S_{q}\right]=R_{0} \otimes B_{m}$ is a commutative ideal $I_{q}$ of $S_{0}$. By Lemma 5.1(iv) we see that $S_{0}=I_{q}+I_{q-1}$; in particular, $S_{0}$ is at most two-step nilpotent.

As $S_{0}$ is irreducible on $S_{-q}$ the central ideal $I=I_{q} \cap I_{q-1}$ acts as a scalar on $S_{-q}$. Since $S_{0}$ is faithful on $S_{-q}$ we must have $\operatorname{dim} I \leqslant 1$. As both $I_{q}$ and $I_{q-1}$ contain a central element of $D_{0}$ we see that $\operatorname{dim} I=1$.

Now we see that $\left[I_{q}, I_{q-1}\right] \subseteq I_{q} \cap I_{q-1}=I$. Therefore [ , ]: $\left(I_{q} / I\right) \times\left(I_{q-1} / I\right) \rightarrow$ $I \simeq k$ defines a pairing. This pairing is nondegenerate. Indeed, if say, $J \subseteq I_{q}$, $\left[J, I_{q-1}\right]=0$, then $J$ is a central ideal of $S_{0}$ and therefore acts by scalars on $S_{-q}$. Therefore $J \subseteq I$. Therefore $n=m$, $\operatorname{dim} S_{0}=2 p^{n}-1$, and $S_{0}$ is a Heisenberg Lie algebra.

It is known (say by [13, Theorem 1]) that all faithful irreducible representations of a Heisenberg algebra of dimension $2 d+1$ are of dimension $p^{d}$. Therefore we must have $\operatorname{dim} S_{-q}=p^{p^{n}-1}$. But $\operatorname{dim} S_{-q}=p^{n}$. So we have the equation $p^{p^{n}-1}=p^{n}$, i.e., $p^{n}-1=n$, which is impossible.

5.9 Corollary. (i) $q \geqslant 8$;

(ii) $\left[S_{q}, S_{-q+1}\right]=0$;

(iii) $\left[\left[S_{q}, S_{-q}\right] S_{-q+1}\right]=0$;

(iv) $\left[\left[S_{q}, S_{-q}\right] V\right]=0$ for every irreducible $D_{0}$-submodule $V$ of $S_{q-1}$.

Proof. Since $p \mid q-1$ and $p \geqslant 7$ we have (i). By Proposition 5.6(iii) (case $j=1, p \mid q-j)$ we have $\left[e_{q}, S_{-q+1}\right]=0$. Thus $0 \neq\left\{x \in S_{q} \mid\left[x, S_{-q+1}\right]=0\right\}$. Since $S_{q}$ is irreducible (by Proposition 5.5(i)), $\left\{x \in S_{q} \mid\left[x, S_{-q+1}\right]=0\right\}=S_{q}$, whence (ii). Now (iii) is evident since $\left[S_{-q+1}, S_{-q}\right]=\left[S_{-q+1}, S_{q}\right]=0$. Finally, (iv) holds in view of Lemma 5.2(ii) since $\left[S_{q}, S_{-q}\right]$ is a commutative ideal of $D_{0}$.

5.10. Arriving at a contradiction: end of proof.

Write $\tilde{S}_{0}=\left[S_{q}, S_{-q}\right]$. By Corollary 5.9(iii), (iv) and Proposition 5.5(iii) we have $\left[\tilde{S}_{0}, \tilde{S}_{0}\right]=\left[\tilde{S}_{0}, S_{-q+1}\right]=\left[\tilde{S}_{0}, V\right]=0$ (where $V$ is any irreducible $D_{0}$-submodule of $\left.S_{q-1}\right)$. By Lemma 5.1(iv), $S_{0}=\tilde{S}_{0}+\left[S_{-q+1}, V\right]$. The preceding equalities now give $\left[S_{0}, \tilde{S}_{0}\right]=0$. Thus $\tilde{S}_{0}$ is a central ideal of $S_{0}$. As $S_{0}$ acts irreducibly on $S_{-q}$ it follows that $\tilde{S}_{0}$ must act by scalars whence $m=1$, i.e., $\operatorname{dim} S_{-q}=1$ (by Proposition 5.5(i)). Since $S_{0}$ is faithful on $S_{-q}$ (by Lemma 5.2(i) and Corollary 5.4) we have $\operatorname{dim} S_{0}=1$.

Now [, ]: $S_{-1} \times S_{1} \rightarrow S_{0}$ defines a pairing of $S_{-1}$ and $S_{1}$. This pairing is nondegenerate since both $S_{-1}$ and $S_{1}$ are $\bar{D}_{0}$-irreducible, where $\bar{D}_{0}=\operatorname{Ker} D_{0} \mid S_{-q}$ (since $D_{0}=S_{0} \oplus \bar{D}_{0}$ ). Suppose that $\operatorname{dim} S_{-1}=n>1$. Let $E_{1}, \ldots, E_{n}$ be a basis of $S_{-1}$, let $F_{1}, \ldots, F_{n}$ be the dual basis of $S_{1}$, and let $S_{0}=k H$. Then we have $\left[E_{i}, F_{j}\right]=$ $\delta_{i j} H$ and $\left[H, E_{i}\right]=E_{i},\left[H, F_{j}\right]=-F_{j}$. Therefore we can apply V. Kac [5, Lemma 2.1 or 2.3]. It says that if $n>1, S$ is infinite-dimensional. Thus $n=1$. But then $S_{-2}=S_{2}=0$, i.e., $S$ is of type $A_{1}$, a contradiction with the assumption that $S$ is a minimal counterexample.

PART III. APpendix: SOME PRoperties of Z - GRADEd Lie Algebras

In this part we collect some results which were used in Part II and which seem to have potential for further applicability. We also include some results which seem to 
go naturally with those we needed in Part II. Finally, we include a result on "asymmetry" between depth and height of a graded Lie algebra. This simple result is used only tangentially, but seems to be applicable and goes well with other results of this part.

We continue to assume that $k$ is algebraically closed of characteristic $p>5$.

1. General graded Lie algebras. We start by recording for future reference a list of different statements (compare [12]). Let $G=\bigoplus_{i=-q}^{r} G_{i}$ be a graded Lie algebra.

g1. $\left[G_{i}, G_{j}\right] \subseteq G_{i+j}$.

g2. $\left[G_{i}, G_{-1}\right]=G_{i-1}$ for $i<0$.

g3. For any $x \in G_{i}, x \neq 0, i>0$, one has $\left[x, G_{-1}\right] \neq 0$.

g4. $G_{-1}$ is an irreducible $G_{0}$-module.

g5. $G_{-1}$ is a faithful $G_{0}$-module.

g6. For any $x \in G_{i}, x \neq 0, i<0$, one has $\left[x, \oplus_{j>0} G_{j}\right] \neq 0$ (i.e., $M(G)=0$ ).

We set $\tilde{G}=\bigoplus_{i \neq 0} G_{i}+\sum_{i \neq 0}\left[G_{i}, G_{-i}\right]$.

1.1. LEMMA. If $\mathrm{gl}$ and $\mathrm{g} 2$ hold in $G$, then $\left[G_{i}, G_{-i}\right] \subseteq\left[G_{1}, G_{-l}\right]$ for any $i \neq 0$.

Proof. We can assume that $i>0$. Our statement is true for $i=1$. Suppose it is true for $i \leqslant m$. Then we have

$$
\begin{aligned}
{\left[G_{m+1}, G_{-m-1}\right] } & =\left[G_{m+1}\left[G_{-m}, G_{-1}\right]\right] \subseteq\left[G_{-m}\left[G_{m+1}, G_{-1}\right]\right]+\left[\left[G_{m+1}, G_{-m}\right] G_{-1}\right] \\
& \subseteq\left[G_{-m}, G_{-m}\right]+\left[G_{1}, G_{-1}\right] \subseteq\left[G_{1}, G_{-1}\right]
\end{aligned}
$$

(We used g2, the Jacobi identity, g1, and inductive assumptions.)

1.2. Proposition. Suppose that $\mathrm{g} 1, \mathrm{~g} 2$ and $\mathrm{g} 3$ hold in $G$. For $V \subseteq G_{i}, i<0$, such that $\left[G_{0}, V\right] \subseteq V,\left[G_{-1}, V\right]=0$, set $H_{i}^{\prime}=V, H_{i+t}^{\prime}=\sum_{0<j \leqslant t}\left(\operatorname{ad} G_{j}\right) H_{i+t-j}^{\prime}$ and $H^{\prime}=$ $\oplus H_{i}^{\prime}$. Then $H^{\prime}$ is an ideal of $G$.

Proof. The proof of [12, Lemma 4.5.1] goes through in our case with only notational differences and gives that $H^{\prime}$ is an ideal of $G$.

1.3. Corollary. Suppose that g1, g2, g3, g4, g5 and g6 hold in $G$. Then $H_{i}^{\prime}=G_{i}$ for $i<0$. In particular:

(i) for any $x \in G_{i}, x \neq 0, i>-q$, one has $\left[x, G_{-1}\right] \neq 0$;

(ii) $G_{-q+j}=\sum_{\alpha_{1}>0, \sum \alpha_{1}=j}\left(\operatorname{ad} G_{\alpha_{1}}\right) \cdots\left(\operatorname{ad} G_{\alpha_{s}}\right) G_{-q}, 0<j<q$;

(iii) $G_{-q}=\left[G_{-q}\left[G_{1}, G_{-1}\right]\right]$;

(iv) $G_{-q}$ is irreducible for $G_{0}$.

Proof. Let $x \in H_{i}^{\prime}$. Applying $\operatorname{ad}\left(\oplus_{j>0} G_{j}\right)$ to $x$ repeatedly we can, by g6, obtain $y \in H_{j}^{\prime}, y \neq 0, j \geqslant 0$. Repeated application of ad $G_{-1}$ to $y$ produces, by $\mathrm{g} 3$ and $\mathrm{g} 5$, a $z \in G_{-1}, z \neq 0$. By Proposition 1.2, $z \in H^{\prime}$. Thus $H_{-1}^{\prime} \neq 0$. By Proposition 1.2, $\left[G_{0}, H_{-1}^{\prime}\right] \subseteq H_{-1}^{\prime}$. Then $\mathrm{g} 4$ gives $H_{-1}^{\prime}=G_{-1}$, whence, by g2, $H_{i}^{\prime}=G_{i}$ for $i<0$.

Now (i) holds for $i \geqslant 0$ by g3, g5. Suppose it does not hold for some $i<0$. Then we can construct $H^{\prime}$ from $V=\left\{x \in G_{i} \mid\left[x, G_{-1}\right]=0\right\}$ and $H_{i}^{\prime}=V$. Therefore $V=G_{i}$. But then $G_{i-1}=\left[V, G_{-1}\right]=0$. Therefore $i=-q$ in view of $\mathrm{g} 2$. This proves (i). 
The right-hand side of (ii) is exactly the definition of $H_{-q+j}^{\prime}$. So (ii) just says that $H_{-q+j}^{\prime}=G_{-q+j}$ which is already established. Next we have $\left[G_{-q}\left[G_{-1}, G_{1}\right]\right]=$ $\left[G_{-1}\left[G_{-q}, G_{1}\right]\right]$. By (ii), with $j=1$, we know that $\left[G_{-q}, G_{1}\right]=G_{-q+1}$. Thus $\left[G_{-q}\left[G_{1}, G_{-1}\right]\right]=\left[G_{-1}, G_{-q+1}\right]=G_{-q}$ as required.

Suppose that $G_{-q}$ is not irreducible. Take $V \subset G_{-q}, V \neq G_{-q},\left[V, G_{0}\right] \subseteq V$. Then $\left[V, G_{-1}\right]=0$ and so $V$ satisfies the assumptions of Proposition 1.2. Then $H_{-q}^{\prime}=V$, a contradiction with the fact that $H_{-q}^{\prime}=G_{-q}$.

1.4. Proposition. Suppose that g1, g2, g3 and g6 hold in G. Assume, in addition, that $G_{1}$ is irreducible and $\left[G_{-q}, G_{1}\right] \neq 0$. Then for any $x \in G_{i}, i>0$, one has $\left[x, G_{-q}\right] \neq 0$.

REMARK. The second condition, $\left[G_{-q}, G_{i}\right] \neq 0$, follows from $\mathrm{g} 4$ and $\mathrm{g} 5$ in view of Corollary 1.3(ii) or (iii).

Proof. Set $V_{i}=\left\{x \in G_{i} \mid\left[x, G_{-q}\right]=0\right\}$. Then $V_{i}$ is a $G_{0}$-submodule of $G_{i}$. Since $G_{1}$ is irreducible and $\left[G_{-q}, G_{1}\right] \neq 0$ by assumptions, we have $V_{1}=0$. Now assume by induction that $V_{i}=0$ for $i \leqslant m, m>0$. Let $x \in V_{m+1}$. Then $0=\left[G_{-1}\left[G_{-q}, x\right]\right]=$ $\left[G_{-q}\left[G_{-1}, x\right]\right]$. Thus $\left[G_{-1}, x\right] \subset V_{m}$. Since $V_{m}=0$, we have $\left[G_{-}, x\right]=0$, whence, by g3, $x=0$. Thus $V_{m+1}=0$, as claimed.

1.5. Proposition. Assume that g1, g2, g3, g4, g5 and g6 hold in G. Assume, in addition, that $\left[G_{-q}, x\right] \neq 0$ for any $x \in G_{q-1}, x \neq 0$. Let $V \subseteq G_{-q+1}, V \neq 0$, and $\left[V, G_{0}\right] \subseteq V$. Then:

(i) $\left[V, G_{-1}\right]=G_{-q}$;

(ii) for any $y \in G_{-1}, y \neq 0$, one has $[V, y] \neq 0$;

(iii) if $q>1$, then for any $x \in G_{q-1}, x \neq 0$, one has $\left[G_{-q}[V, x]\right] \neq 0$;

(iv) if $q>1$, then for any $x \in G_{q-1}, x \neq 0$, one has $[V, x] \neq 0$.

Proof. First, (i) follows from Corollary 1.3(i), (iv). To prove (ii) note that $U=\left\{y \in G_{1} \mid[y, V]=0\right\}$ is a $G_{0}$-submodule of $G_{-1}$. Corollary 1.3(i) implies that $U \neq G_{-1}$ and then $\mathrm{g} 4$ implies that $U=0$, whence (ii). To prove (iii) observe that $\left[G_{-q}[V, x]\right]=\left[V\left[G_{-q}, x\right]\right]$. Now $\left[G_{-q}, x\right] \neq 0$ by assumption. Since $\left[G_{-q}, x\right] \subseteq G_{-1}$ we get (iii) from (ii). Finally, (iv) follows directly from (iii).

1.6. Lemma. Suppose that g1, g2, g3, g4, g5 and $\mathrm{g} 6$ hold in G. Assume, in addition, that $q>1$ and $\left[G_{-q}, x\right] \neq 0$ for any $x \in G_{q-1}, x \neq 0$. Let $U \neq 0$ be a $G_{0}$-submodule of $G_{q-1}$. Then $[y, U] \neq 0$ for any $y \in G_{-q+1}, y \neq 0$.

Proof. We have $\left[G_{-q}, U\right]=G_{-1}$ in view of $\mathrm{g} 4$ and since $\left[G_{-q}, U\right] \neq 0$ by assumption. Therefore $\left[G_{-q}[y, U]\right]=\left[y,\left[G_{-q}, U\right]\right]=\left[y, G_{-1}\right]$ and the latter is not zero by Corollary 1.3(i).

1.7. Proposition. Suppose that g1, g2, g3, g4, g5 and g6 hold in G. Assume, in addition, that $q>1$ and $\left[G_{-q}, x\right] \neq 0$ for any $x \in G_{q-1}, x \neq 0$. Let $V \neq 0$ be $a$ $G_{0}$-submodule of $G_{q-1}$. Then:

(i) $[y, V] \neq 0$ for any $y \in G_{-q+1}, y \neq 0$;

(ii) $\left[G_{1}, G_{-1}\right] \subseteq\left[G_{-q}, G_{q}\right]+\left[G_{-q+1}, V\right]$. 
Proof. Note first that $\left[G_{-q}, V\right] \neq 0$ by assumption. Therefore $\left[G_{-q}, V\right]=G_{-1}$ by g4. Therefore $\left[G_{-q}[y, V]\right]=\left[y\left[G_{-q}, V\right]\right]=\left[y, G_{-1}\right]$ and now (i) follows from Corollary 1.3(i). Next,

$$
\begin{aligned}
{\left[G_{1}, G_{-1}\right] } & =\left[G_{1}\left[G_{-q}, V\right]\right] \subseteq\left[G_{-q}\left[G_{1}, V\right]\right]+\left[\left[G_{1}, G_{-q}\right] V\right] \\
& \subseteq\left[G_{-q}, G_{q}\right]+\left[G_{-q+1}, V\right]
\end{aligned}
$$

as asserted.

1.8. Lemma. Suppose that g1 and g2 hold in $G$. Let $V \subseteq G_{1}$ and $\left[V, G_{0}\right] \subseteq V$. Set $H_{i}=G_{i}$ for $i \leqslant 0, H_{1}=V$ and $H_{i+1}=\left\{x \in G_{i+1} \|\left[G_{-1}, x\right] \subseteq H_{i}\right\}, i>0$. Then $H=$ $\oplus H_{i}$ is a subalgebra of $G$.

Proof. We have $\left[H_{i}, H_{j}\right] \subseteq H_{i+j}$ for $i, j \leqslant 0$ by g1. By construction, $\left[H_{i}, H_{-1}\right]=$ $\left[H_{i}, G_{-1}\right] \subseteq H_{i-1}$ for any $i$. Therefore, $\left[H_{i}, H_{j}\right] \subseteq H_{i+j}$ for any $i$ and any $j<0$. It is easy to check that $\left[H_{0}, H_{i}\right] \subseteq H_{i}$. Suppose by induction that $\left[H_{i}, H_{j}\right] \subseteq H_{i+j}$ if $i+j \leqslant m$. Then

$$
\left[H_{-1}\left[H_{i}, H_{m+1-i}\right]\right] \subseteq\left[H_{i-1}, H_{m+1-i}\right]+\left[H_{i}, H_{m-i}\right] \subseteq H_{m}+H_{m} \subseteq H_{m},
$$

whence $\left[H_{i}, H_{m+1-i}\right] \subseteq H_{m+1}$ by definition of $H_{i}, i>0$.

1.9. Proposition. Suppose that $\mathrm{g} 1, \mathrm{~g} 2$ and $\mathrm{g} 3$ hold in G. Assume, in addition, that $G_{i+1}=\left[G_{1}, G_{i}\right]$ for $i>0$. Let $V$ be a maximal $G_{0}$-submodule of $G_{1}$, and let $H$ be the algebra constructed from $V$ as in Lemma 1.8. Then:

(i) $H$ is a maximal subalgebra of $G$;

(ii) $G_{1} / V$ is contained in every nontrivial $H$-submodule of $G / H$.

Proof. Let us first prove (ii). Let $\bar{W} \neq 0$ be an $H$-submodule of $G / H$, and let $W$ be its preimage in $G$. So $W \supseteq H, W \neq H$ and $[W, H] \subseteq W$. Take $x \in W$ and let $m$ be the largest integer such that $\left(\operatorname{ad} G_{-1}\right)^{m} x \not \subset H$. Take $y \in\left(\operatorname{ad} G_{-1}\right)^{m} x, y \notin H$. Write $y=\oplus y_{i}$. Then $\left[G_{-1}, y_{i}\right] \subseteq H_{i-1}$ for all $i$. For $i \leqslant 0$ we have $H_{i}=G_{i}$, whence $y_{i} \in H_{i}$. For $i>1$ the condition $\left[G_{-1}, y_{i}\right] \subseteq H_{i-1}$ means that $y_{i} \in H_{i}$ (by construction of $H_{i}$ ). Thus $y \equiv y_{1} \bmod H$. Since $y \notin H$ it means that $y_{1} \notin V$. Since $G_{1} / V$ is irreducible it means that $G_{1} \subseteq W$; that is, $G_{1} / V \subseteq \bar{W}$. This proves (ii).

To prove (i) take a subalgebra $M \supset H, M \neq H$. Then $[M, H] \subseteq M$, whence, by (ii), $M \supseteq G_{1}$. Since $G_{i+1}=\left[G_{i}, G_{1}\right]$ for $i>0$, it means that $M=G$. This proves (i).

1.10. Lemma. Assume that $\mathrm{g} 1, \mathrm{~g} 2, \mathrm{~g} 3$ and $\mathrm{g} 4$ hold in $G$. Let $x$ be a nilpotent element of $G_{0}\left(\right.$ i.e, $\left.(\operatorname{ad} x)^{\operatorname{dim} G_{0}} G_{0}=0\right)$. Then $x$ acts as $i \lambda \cdot \mathrm{Id}+($ nilpotent operator $)$ on $G_{i}$.

Proof. Our statement is true by assumption for $i=0$. Let (see Lemma I.1.3) $m$ be such that $n=x^{p^{m}}$ is in the center of the universal enveloping algebra $U$ of $G_{0}$. We extend action of $G_{0}$ on $G$ to the action of $U$. Then $n$ acts as a scalar, say $\mu$, on $G_{-1}$ by $\mathrm{g} 4$. Therefore, by $\mathrm{g} 2$ it acts as $i \mu$ on $G_{i}, i<0$. Of course, $n$ acts as 0 on $G_{0}$. Using $\mathrm{g} 3$ we establish successively that $n$ acts as $i \mu$ on all $G_{i}$. Set $\lambda=\sqrt[p^{m}]{\mu}$. Then $x$ acts as $i \lambda \cdot I d+$ (nilpotent operator) on $G_{i}$, as asserted. 
1.11. Corollary. Assume that $\mathrm{g} 1, \mathrm{~g} 2, \mathrm{~g} 3, \mathrm{~g} 4$ and $\mathrm{g} 5$ hold in $G$. Let I be a nilpotent ideal of $G_{0}$. Then:

(i) if $p+i, I$ acts faithfully on $G_{i}$ and $[I, V]=V$ for every $G_{0}$-submodule of $G_{i}$;

(ii) $I$ acts nilpotently on $G_{p i}$ and it acts trivially on every simple $G_{0}$-submodule $V$ of $G_{p i}$.

Proof. Since $I$ is a nilpotent ideal of $G_{0}$, every element of $I$ is a nilpotent element of $G_{0}$. By Lemma 1.10 there exists a function $\lambda: I \rightarrow k$ such that $x \in I$ acts as $i \lambda(x)$ Id + (nilpotent operator) on $G_{i}$. This directly implies (ii). Let $J$ be the kernel of the action of $I$ on a $G_{0}$-submodule $V \subseteq G_{i}$. Then $J$ is a nilpotent ideal. If $p+i$ we see that $\lambda(J)=0$, i.e. $J$ acts nilpotently on $G_{-1}$. Since $G_{-1}$ is irreducible it means that $\left[J, G_{-1}\right]=0$ in contradiction with g5. This proves (i).

1.12. Proposition. Suppose that $\tilde{G}$ is simple and that $\mathrm{g} 1$ and $\mathrm{g} 2$ hold in $G$. Then:

(i) g3, g5 and g6 hold in $G$;

(ii) $\tilde{G}_{0}=\left[G_{1}, G_{-1}\right]$;

(iii) $G_{-q}$ is irreducible for $\left[G_{1}, G_{-1}\right]$;

(iv) $\left[G_{-q}\left[G_{1}, G_{-1}\right]\right]=G_{-q}$;

(v) $G_{-q+i}=\sum_{\alpha_{s}>0, \sum \alpha_{1}=i}\left(\operatorname{ad} G_{\alpha_{1}}\right) \cdots\left(\operatorname{ad} G_{\alpha_{s}}\right) G_{-q}$ for $i>0$;

(vi) if $G_{1}$ is irreducible, then $x \in G_{i}, i>0,\left[x, G_{-q}\right]=0$ implies that $x=0$.

Proof. We have (ii) by Lemma 1.1. Now (iii) and (v) are contained in [12, Lemma 4.5.1(ii) and (iii)]. Again by [12, Lemma 4.5.1(i)], g3 and g5 hold in $G$. If g6 does not hold, then $M(G) \neq 0$ (see $[12, \S 1.5]$ ). But $M(G)=0$ since $\tilde{G}$ is simple. Thus g6 holds. This proves (i). Now Proposition 1.4 is applicable and gives (vi). Finally, we have $\left[G_{-1}, G_{-q+1}\right]=G_{-q}$ (by g2) and $\left[G_{1}, G_{-q}\right]=G_{-q+1}$ (by (v)). Therefore $\left[G_{-q}\left[G_{1}, G_{-1}\right]\right]=\left[\left[G_{-q}, G_{1}\right] G_{-1}\right]=\left[G_{-q+1}, G_{-1}\right]=G_{-q}$, whence (iv).

2. On asymmetry of graded Lie algebras. The purpose of this section is to establish

2.1. TheOREM. Let $G=\bigoplus_{i=-q}^{r} G_{i}$. Suppose that $G$ satisfies $\mathrm{g} 4$ and $\mathrm{g} 5$, and that:

(a) $\tilde{G}$ is simple;

(b) for any $x \in G_{i}, i>0,\left[x, G_{-q}\right]=0$ implies $x=0$;

(c) for any $x \in G_{i}, i<0,\left[x, G_{r}\right]=0$ implies $x=0$;

(d) $\tilde{G}$ is generated by $G_{1}$ and $G_{-1}$.

Then either $\tilde{G}$ is a classical Lie algebra or $\max (q, r) \geqslant((p-1) / 2) \min (q, r)$.

2.1.1. REMARK. The conditions of Theorem 2.1 are satisfied if $\tilde{G}$ is simple, $G_{-1}$ and $G_{1}$ are irreducible and faithful $G_{0}$-modules, and $\tilde{G}$ is generated by $G_{1}$ and $G_{-1}$. Indeed, in this case Proposition 1.12(vi) applied both to $G$ and to $G$ with inverted grading gives the desired properties.

2.1.2. REMARK. The condition $\max (q, r)<((p-1) / 2) \max (q, r)$ is equivalent to two inequalities: $2 r<p q-q$ and $2 q<p r-r$.

Indeed, these inequalities can be written as $2(r+q)<p q+q, 2(r+q)<p r+r$, or $2(r+q)<(p+1) \min (q, r)$. But $r+q=\max (q, r)+\min (q, r)$. That is,

$$
2 \max (q, r)+2 \min (q, r)<(p+1) \min (q, r),
$$


or

$$
\max (q, r)<((p-1) / 2) \min (q, r)
$$

as required.

2.1.3. REMARK. This part of the paper draws heavily on [14] although more in spirit than in results.

2.2. Lemma. (i) If $2 r<p m-q, m>0$, then $\exp \operatorname{ad} G_{-j}, j \geqslant m$, exists and consists of automorphisms of $G$.

(ii) If $2 q<p n-r, n>0$, then $\exp \operatorname{ad} G_{j}, j \geqslant n$, exists and consists of automorphisms of $G$.

Proof. We prove only (i), as (ii) is proved in the same way. For $\exp \operatorname{ad} G_{-j}$ to exists one must have $\left(\operatorname{ad} G_{-j}\right)^{p}=0$. But $\left(\operatorname{ad} G_{-j}\right)^{p} G \subseteq \oplus_{i} G_{i-p j}$. Since $j \geqslant m$, we have $2 r<p j-q$, i.e., $r-p j<-r-q<-q$, whence $\oplus_{i} G_{i-p j}=0$.

Next, in view of [14, Lemma 1.2] we know that exp ad $x$ is an automorphism if $h_{u !:}=\left[(\operatorname{ad} x)^{\alpha} y,(\operatorname{ad} x)^{\beta} z\right]=0$ for all $y, z \in G, \alpha+\beta \geqslant p$. Take $x \in G_{-j}, y \in G_{s}$, $z \in G_{t}, \alpha+\beta \geqslant p$. Then $h_{\alpha \beta} \in G_{-j(\alpha+\beta)+s+r}$. But $-j(\alpha+\beta)+s+t \leqslant-m p+2 r$ $<-q$, whence $h_{\alpha \beta}=0$. This concludes the proof of (i).

2.3. Proposition. Suppose that $\tilde{G}$ has no center and $2 r<p m-q, 2 q<p n-r$ with $m>0, n>0$. Then the subalgebra $H$ of $G$ generated by $H_{1}=\bigoplus_{i \leqslant-m} G_{i}$ and $H_{2}=\oplus_{1 \geqslant n} G_{i}$ is, modulo its radical, a direct sum of simple classical Lie algebras (we say simply: classical). Moreover, if Nilrad $H$ is the maximal ideal of $H$ acting nilpotently on $G$ then $H / \mathrm{Nilrad} H$ has only central radical and $H / \mathrm{Nilrad} H$ coincides with its derived algebra.

Proof. Since $\tilde{G}$ has no center we have an imbedding ad: $\tilde{G} \rightarrow \operatorname{Der} \tilde{G}$. We identify subsets of $\tilde{G}$ with their images under ad. Consider the subgroup $\mathscr{H}$ of Aut $\tilde{G}$ generated by exp ad $H_{1}$ and expad $H_{2}$ (meaningful by Lemma 2.2). For the Lie algebra of $\mathscr{H}$, Lie $\mathscr{H}$, we have Lie $\mathscr{H} \subseteq \operatorname{Der} G$ and Lie $\mathscr{H} \supseteq H_{1} \oplus H_{2}$. Therefore Lie $\mathscr{H} \supseteq H$. Clearly, $H$ is stable under $\mathscr{H}$ and therefore $H$ is an ideal of Lie $\mathscr{H}$. Let $\mathscr{N}$ be the unipotent radical of $\mathscr{H}$. Then $\mathscr{H} / \mathscr{N}$ is reductive and generated by unipotent subgroups exp ad $H_{i}(\bmod \mathscr{N}), i=1,2$. Therefore $\mathscr{H} / \mathscr{N}$ is semisimple. Write $\mathscr{H} / \mathscr{N}$ as an almost direct product of factors $\mathscr{S}_{i}$ such that all simple factors of $\mathscr{S}_{i}$ are of the same type. Write $S_{i}=$ Lie $\mathscr{S}_{i}$. If simple factors of an $\mathscr{S}_{i}$ are not of type $A_{p t-1}$, then $S_{i}$ is a direct sum of simple Lie algebras. Otherwise $S_{i}$ may have center and may be distinct from their derived algebras. However, since $S_{i} \cap H /(H \cap$ Lie $\mathscr{N})$ is generated by nilpotent elements, we always have that $S_{i} \cap H /(H \cap \operatorname{Lie} \mathscr{N})$ coincides with its derived algebra. Since $H \cap$ Lie $\mathscr{N}$ is an ideal of $H$ which acts nilpotently on $G$, our proposition is proven.

2.4. Corollary. Take assumptions and notation of Proposition 2.3 and assume, in addition, that $H$ is not nil. Then $q \geqslant n, r \geqslant m$.

Proof. Let $\mathscr{T}$ be the one-dimensional subtorus of Aut $\tilde{G}$ defining the grading of $\tilde{G}$ (see $[12, \S 1.4])$. Then $\mathscr{T}$ normalizes $H$ and $\mathscr{H}$. Since $\mathscr{T}$ preserves Nilrad $H$, it acts on 
$H /$ Nilrad $H$. By condition $H /$ Nilrad $H \neq 0$, it is classical. But in a classical Lie algebra any Z-grading is symmetric (i.e., it has height equal to depth). Since $H /$ Nilrad $H$ is generated by images of $H_{1}=\oplus_{i=-q}^{-m} G_{i}, H_{2}=\oplus_{i=n}^{r} G_{i}$, this implies that $q \geqslant n, r \geqslant m$.

2.5. Lemma. Take assumptions of Theorem 2.1. Then the subalgebra $H$ of $G$ generated by $G_{-q}$ and $G_{r}$ is not nil.

Proof. Suppose it is. Let $\mathscr{T}$ be the one-dimensional torus defining the grading of $G$. Since $H$ is invariant under $\mathscr{T}$ so is its center $C$. So $C=\oplus C_{i}$. By conditions (b) and (c) of Theorem 2.1 we have that $C=C_{0}$, i.e., $C \subseteq G_{0}$. But then $C$ is an ideal of $G_{0}$. Since the action of $C$ on $\tilde{G}$ is nilpotent and in view of $\mathrm{g} 4$ and g5 we have $C=0$, a contradiction, since a nilpotent algebra must have a nontrivial center.

2.6. Proof of Theorem 2.1. Suppose $\max (q, r)<((p-1) / 2) \min (q, r)$. By Remark 2.1.2 this is equivalent to $2 r<p q-q, 2 q<p r-r$. By Lemma 2.5 we know that $H=\left\langle G_{-q}, G_{r}\right\rangle$ is not nil. Therefore we can apply Corollary 2.4 with $m=q, n=r$. It gives us $q \geqslant r, r \geqslant q$; that is, $q=r$.

If $q=1$ then we are done by Proposition 2.3 (since $H=\tilde{G}$ is simple). If $q \geqslant 2$, we consider $H_{1}=G_{-q} \oplus G_{-q+1}$ and $H_{2}=G_{q} \oplus G_{q-1}$. We have $p(q-1)-q=$ $(p-1) q-p>2 q$ if $p \geqslant 7$ (and also if $q>2, p \geqslant 5$ ). Therefore we can apply Proposition 2.3 (with $m=n=q-1$ ). In this case $H=\left\langle H_{1}, H_{2}\right\rangle$ contains $\left[G_{-q}, G_{q-1}\right]=G_{-1},\left[G_{q}, G_{-q+1}\right]=G_{1}$ and since $\tilde{G}$ is generated by $G_{1}$ and $G_{-1}$ we have $H=\tilde{G}$. So Proposition 2.3 says that $\tilde{G}$ is classical.

ACKNOWLeDGements. I am grateful to Richard Block, Victor Kac and Robert Wilson for suggesting numerous corrections and improvements and for otherwise pleasant and useful conversations. In particular, Robert Wilson has corrected the proof of Theorem I.5.1.

\section{REFERENCES}

1. R. E. Block, Modules over differential polynomials rings, Bull. Amer. Math. Soc. 79 (1973), 729-733.

2.

3. Determination of the differentiably simple rings with minimal ideal, Ann. of Math. (2) 90 (1969), 433-459.

4. M. Frank, A new simple Lie algebra of characteristic three, Proc. Amer. Math. Soc. 38 (1973), 43-46.

5. V. Kac. On the classification of the simple Lie algebras over fields with non-zero characteristic, Math. USSR-Izv. 4 (1970), 391-413.

6. __ The simple irreducible graded Lie algebras of finite growth. Izv. Akad. Nauk SSSR Ser. Mat. 32 (1968), 1323-1367.

7. Automorphisms of finite order of semi-simple Lie algebras, J. Funct. Anal. Appl. 3 (1969), 252-254. (For a detailed exposition, see S. Helgason, Differential geometry, Lie groups and symmetric spaces, Academic Press, New York, 1978.)

8. I. L. Kantor, Graded Lie algebras, Trudy Seminara po Vectornomu y Tensornomu Analyzu, No. XV. Moscow State Univ., 1970, pp. 227-266. (See also a short summary in Soviet Math. Dokl. 9 (1968), 409-412.)

9. M. I. Kuznetsov. The modular simple Lie algebras with a solvable maximal subalgebra, Math. USSR-Sb. 30 (1976), 68-76.

10. R. Ree, On generalized Witt algebras. Trans. Amer. Math. Soc. 83 (1956), 510-546.

11. J. R. Schue, Cartan decompositions for Lie algebras of prime characteristic, J. Algebra 11 (1969), 25-52; Erratum 13 (1969), 558. 
12. B. Weisfeiler, On the structure of the minimal ideal of some graded Lie algebras in characteristic $p>0$. J. Algebra 53 (1978), 344-361.

13. B. Weisfeiler and V. Kac, The irreducible representations of Lie p-algebras, Funct. Anal. Appl. 5 111-117.

14. __ Exponentials in Lie algebras of characteristic p, Math. USSR-Izv. 5 (1971), 777-803.

15. R. L. Wilson, Classification of generalized Witt algebras over an algebraically closed field, Trans. Amer. Math. Soc. 153 (1971), 191-210.

16. H. Zassenhaus, The representations of Lie algebras of prime characteristic, Proc. Glasgow Math. Assoc. 2 (1954), 1-36.

Department of Mathematics, Pennsylvania State University, University Park, Pennsylvania 16802 